\title{
Linear stability analysis and numerical simulation of miscible two-layer channel flow
}

\author{
K. C. Sahu, H. Ding, P. Valluri and O. K. Matar* \\ Department of Chemical Engineering, \\ Imperial College London, SW7 2AZ, UK \\ (Dated: February 9, 2009)
}

\begin{abstract}
The stability of miscible two-fluid flow in a horizontal channel is examined. The flow dynamics are governed by the continuity and Navier-Stokes equations coupled to a convective-diffusion equation for the concentration of the more viscous fluid through a concentration-dependent viscosity. Our analysis of the flow in the linear regime delineates the presence of convective and absolute instabilities and identifies the vertical gradients of viscosity perturbations as the main destabilizing influence in agreement with previous work. Our transient numerical simulations demonstrate the development of complex dynamics in the nonlinear regime, characterized by roll-up phenomena and intense convective mixing; these become pronounced with increasing flow rate and viscosity ratio, as well as weak diffusion.
\end{abstract}

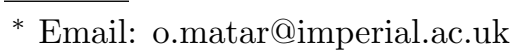




\section{INTRODUCTION}

The stability of two-layer flows in planar channels and pipes has received considerable attention in the literature experimentally, theoretically and numerically. This is due to the central importance of these flows to numerous engineering applications. In the oil and gas industry, the transportation of crude oil in pipelines relies on the stability of two-layer flows when the less viscous fluid is at the wall [1]. In the chemical process industry, the stability of two-layer flows features in centreline injectors, which are used upstream of static mixers, employed for mixing liquids [2]. In the food and drink industry, cleaning of plants, involves the removal of a highly viscous fluid by fast-flowing water pumped through the plant. In dairy plants, flows involving these displacements are used during plant start-up and rinsing [3]. Achieving fundamental understanding of these flows in the latter context is important in order to determine the degree of mixing between the fluids and to minimize the amount of waste-water utilized.

A large number of investigations have focused on the stability of immiscible fluids (see, for instance, $[1,4-10]$ and references therein). Starting with the work of Yih [4] and Hickox [5], carried out using long-wave theory in planar channels and cylindrical pipes, respectively, these studies have shown that two-layer flows are destabilized linearly by an 'interfacial' mode at arbitrarily small Reynolds numbers. This is brought about by a discontinuity in the slope of the base state velocity at the interface $[11,12]$. Short-wave asymptotics were also carried out [13] showing that viscous stratification of two unbounded, immiscible fluids undergoing Couette flow leads to an unconditional instability in the absence of surface tension; the physical mechanism of this instability was elucidated by Hinch [14]. At sufficiently large Reynolds numbers, a short-wave, 'shear' mode also becomes unstable [15]. An extensive review of the work on the linear stability of planar two-fluid Poiseuille flow is provided in [16]. A number of studies have also been carried out on the stability of immiscible core-annular flows in connection with lubricated oil pipelines and static mixers $[1,8]$. These have included linear stability analyses for horizontal [6, 7, 17-19] and vertical pipes [5, 20], accounting for viscosity and density contrasts, experiments [21, 22] and numerical simulations in straight $[9,10,23]$ and corruagated pipes [24-27].

The stability of miscible two-layer flows has received comparatively less attention than that of immiscible systems. Linear stability analyses of three-layer Poiseuille channel flows 
by Ranganathan and Govindarajan [28] and Govindarajan [29] have shown these flows to be unstable at low Reynolds number and high Schmidt number. Other studies involving channel flows have demonstrated the destabilising effect of diffusion for continuous but rapidly varying viscosity stratification [30]. In the case of core-annular miscible flows, some experimental studies have focused on determining the thickness of the more viscous fluid left on the pipe walls following its displacement by a less viscous fluid and on measuring the tip speed of the propagating 'finger' of the latter [31-36]. Others works have examined the development of "interfacial' axisymmetric and "corkscrew" patterns that accompany these flows [2, 37-40]. More recently, axisymmetric "pearl" and "mushroom" patterns were observed experimentally in the case of neutrally-buoyant, miscible core-annular flows in horizontal pipes at high Schmidt number and Reynolds numbers in the range 2-60 [41]. For fixed viscosity ratios, the transition from "pearls" to "mushrooms" occurred with increasing Reynolds and/or the core radius. The most recent work on the linear stability of neutrallybuoyant, core-annular flows was that of Sevlam et al. [42]. These authors show that beyond a critical viscosity ratio, the flow is unstable even when the less viscous fluid is at the wall, in contrast to the case of immiscible lubricated pipelining [1] and to miscible channel flows [43], which are stable in this configuration. This study also shows that axisymmetric (corkscrew) modes are dominant if the more (less) viscous fluid is in the pipe core, and for large Schmidt numbers, relatively small Reynolds number and large wavenumbers.

In this paper, we examine the stability of two-layer miscible flows in planar channels, focusing on the neutrally-buoyant displacement of a highly viscous fluid by a less viscous one at relatively large Reynolds number. These flows are modelled via solution of the Navier-Stokes equations coupled to a convective-diffusion equation for the concentration of the more viscous fluid. A generalized linear stability analysis [44-46] (in which both the spatial wavenumber and temporal frequency are complex) is carried out, which allows the demarcation of the boundaries between convectively and absolutely unstable flows in the space of relevant parameters: the Reynolds and Schmidt numbers, and a viscosity ratio. This analysis is performed for a three-layer flow wherein the channel walls are coated with a layer of the more viscous fluid of uniform thickness. To the best of our knowledge, this type of analysis, which has been performed previously for jets, mixing layers, wakes, boundary layers etc, has not been carried out for miscible channel flows; such an analysis, however, was conducted for immiscible two-layer flows to determine the occurrence of defects in the 
co-extrusion of polymers [47].

The nonlinear stability of these flows is also examined via transient numerical simulations of the fully-nonlinear governing equations; attention is focused on relatively large Reynolds numbers (in the range 100-1000), which has received little attention for miscible two-layer flows in the literature. Two distinct cases are considered: one is the three-layer flow; the other involves channels initially filled completely with the more viscous fluid. The former case allows comparisons between the predictions of linear theory and the numerical simulations to be made. The latter is relevant to the modelling of applications in the food and drink and oil and gas industries, for instance, that involve the complete removal of a highly viscous 'soil' or 'foulant' (e.g. a toothpaste or an asphaltene deposit) from a channel by a less viscous fluid (e.g. water or crude oil).

The rest of this paper is organised as follows. Details of the problem formulation are provided in section II, and the results of the linear stability analysis are presented in section III. In section IV, we discuss the results of our numerical simulations and provide concluding remarks in section $\mathrm{V}$.

\section{FORMULATION AND PROBLEM STATEMENT}

\section{A. Governing equations}

We consider the flow of two miscible, Newtonian and incompressible fluids of equal density and varying viscosity in a horizontal, planar channel. We use a rectangular coordinate system, $(x, y)$, to model this flow where $x$ and $y$ denote the horizontal and vertical coordinates, respectively. The channel walls, which are rigid and impermeable, are located at $y=0$ and $y=H$, while its inlet and exit coincide with $x=0$ and $x=L$, respectively. The flow dynamics are governed by the continuity and Navier-Stokes equations, in addition to a convective-diffusion equation for the concentration of the more viscous fluid. Solutions of these equations are subject to no-slip, no-penetration and no-flux conditions at $y=(0, H)$. We imposed a fully-developed velocity profile with a constant flow rate at the inlet $(x=0)$, and Neumann boundary conditions at the outlet $(x=L)$ in the extended domain simulations. 
We render these equations dimensionless by introducing the following scaling:

$$
\mathbf{u}=(u, v)=V \tilde{\mathbf{u}}=V(\tilde{u}, \tilde{v}), \quad(x, y)=H(\tilde{x}, \tilde{y}), \quad t=\frac{H}{V} \tilde{t}, \quad p=\rho V^{2} \tilde{p}, \quad \mu=\mu_{2} \tilde{\mu}
$$

in which $\mathbf{u}=(u, v)$ represents the two-dimensional velocity field where $u$ and $v$ denote the horizontal and vertical velocity components, and $p, \rho, \mu$ and $t$ denote pressure, density, viscosity and time, respectively; the tildes designate dimensionless quantities. Note that the concentration, which represents the fraction of the channel occupied by the more viscous fluid, is already dimensionless. In Eq. (1), $V \equiv Q / H$ represents a characteristic velocity, in which $Q$ denotes the total flow rate, and the viscosity has been scaled on that of the less viscous fluid, $\mu_{2}$. The dimensionless governing equations are then given by

$$
\begin{gathered}
\nabla \cdot \tilde{\mathbf{u}}=0 \\
\frac{\partial \tilde{\mathbf{u}}}{\partial \tilde{t}}+\tilde{\mathbf{u}} \cdot \nabla \tilde{\mathbf{u}}=-\nabla \tilde{p}+\frac{1}{\operatorname{Re}} \nabla \cdot\left[\tilde{\mu}\left(\nabla \tilde{\mathbf{u}}+\nabla \tilde{\mathbf{u}}^{T}\right)\right], \\
\frac{\partial \tilde{c}}{\partial \tilde{t}}+\tilde{\mathbf{u}} \cdot \nabla \tilde{c}=\frac{1}{\mathrm{Pe}} \nabla^{2} \tilde{c},
\end{gathered}
$$

where $\tilde{c}$ is the concentration, $\operatorname{Re} \equiv \rho V H / \mu_{2}$ and $\mathrm{Pe} \equiv V H / \mathcal{D}$ represent a Reynolds number and a Péclet number, respectively; here, $\mathcal{D}$ denotes a constant diffusion coefficient (even though a concentration-dependent $\mathcal{D}$ can have a non-negligible effect on the stability characteristics [48]). Note that we shall use the Schmidt number, Sc $\equiv$ Pe/Re, to characterise the effect of diffusion on the linear stability characteristics. We shall also take $\tilde{\mu}$ to be an exponential function of $\tilde{c}: \tilde{\mu}=e^{\tilde{c} \ln (m)}[42,49]$ where $m \equiv \mu_{1} / \mu_{2}$ is a viscosity ratio in which $\mu_{1}$ represents the viscosity of the more viscous fluid. In the present work, we focus on the stability of the flow with $m \geq 1$ corresponding to situations in which the less viscous fluid is in the channel interior.

\section{B. Fully-developed, three-layer flow: linear stability}

Here, we consider the base state whose linear stability characteristics will be analyzed. This corresponds to a parallel, fully-developed flow in which the two fluids are separated by a mixed layer of uniform thickness $q$, with the less viscous fluid located in the region $1 / 2-h \leq y \leq 1 / 2+h$, as shown in Fig. 1 . The temporal stability analysis of three-layer flow in channel and pipe has been studied previously in the literature [28, 43, 50-52]. As will 
be discussed in the following section, only half the channel will be considered in which the base state concentration, $C$, is characterized by the following steady concentration profile

$$
\begin{aligned}
& C=0, \quad 1 / 2 \leq y \leq h+1 / 2, \\
& C=\sum_{i=1}^{6} a_{i} y^{i-1}, \quad h+1 / 2 \leq y \leq h+q+1 / 2, \\
& C=1, \quad h+q+1 / 2 \leq y \leq 1,
\end{aligned}
$$

where $a_{i}(i=1,6)$ are given by

$$
\begin{aligned}
& a_{1}=-\frac{h^{3}}{q^{5}}\left(6 h^{2}+15 h q+10 q^{2}\right), \\
& a_{2}=\frac{30 h^{2}}{q^{5}}(h+q)^{2} \\
& a_{3}=-\frac{30 h}{q^{5}}\left(2 h^{2}+3 h q+q^{2}\right), \\
& a_{4}=\frac{10}{q^{5}}\left(6 h^{2}+6 h q+q^{2}\right), \\
& a_{5}=-\frac{15}{q^{5}}(2 h+q), \\
& a_{6}=\frac{6}{q^{5}} .
\end{aligned}
$$

Thus, the concentration of the less and more viscous fluids are equal to 0 and 1 , respectively, in the channel core and adjacent to the channel walls; the concentration in the top and bottom mixed layers are described by fifth order polynomials. The constants $a_{i}(i=1,6)$ are obtained by demanding that the concentration and its first two derivatives be continuous at the edges of the mixed layers. We have also applied symmetric boundary conditions across the channel centerline. It is worthy of mention that very similar results to those that will be discussed in section III were obtained via the use of a hyperbolic tangent profile instead of the polynomial in Eq. (5).

The concentration profile described by Eq. (5) corresponds to an idealized state, achievable for a sufficiently long distance from the inlet and for relatively weak diffusion characterized by large Pe. The quasi-steady base state velocity profile, $U(y)$, is then obtained by solving the fully-developed version of Eq. (3):

$$
\operatorname{Re} P=\frac{d}{d y}\left(e^{C \ln m} \frac{d U}{d y}\right)
$$

where $P$ is a constant pressure gradient. Solutions of Eq. (7) are obtained subject to no-slip and no-penetration conditions at the walls, $y=(0,1)$. The value of $P$ is chosen such that 
the flow rate is constant, $Q=\int_{0}^{1} U d y=1$; this is then consistent with the scaling introduced in Eq. (1). The stability of the steady state characterized by $U$ and $C$ and parameterized by $h, q, m$ and Re will be investigated in section III.

\section{Three-layer and displacement flows: direct numerical simulations}

In the present work, two configurations will be investigated using direct numerical simulations: one is the three-layer flow described in the previous section and shown schematically in Fig. 1; the other corresponds to a flow involving the displacement of the more viscous fluid, which occupies the entire channel initially, by the less viscous one. The imposed boundary conditions are the following. At the channel inlet, the flow rate is kept constant with a Poiseuille channel flow velocity profile, obtained via the integration of Eq. (7):

$$
u=\operatorname{ReP} \int_{0}^{y} \frac{y}{e^{C l n m}} d y+c_{1} \int_{0}^{y} \frac{1}{e^{C l n m}} d y+c_{2}, \quad \text { and } \quad v=0 .
$$

Here, the integration constants, $c_{1}$ and $c_{2}$, are obtained from the no-slip boundary condition at the walls. In the case of the three-layer flows, a periodic oscillation of magnitude $10^{-3}$ is applied to $h$ at the inlet. The concentration profile, given by Eq. (5), is then obtained using the instantaneous $h$ at the inlet. For the displacement flows, $C=0$ in Eq. (8) in order to determine $u$ at the channel inlet. At the channel outlet, we impose outflow conditions, given by $\partial \mathbf{u} / \partial x=0$ and $\partial c / \partial x=0$, for both flows. No-slip, no-penetration and no-flux boundary conditions are enforced at the upper and bottom channel walls for both flows; the latter conditions are expressed by $\mathbf{n} \cdot \nabla c=0$ wherein $\mathbf{n}$ is the unit vector normal to the solid walls.

\section{LINEAR STABILITY ANALYSIS}

\section{A. Linearization and numerical procedure}

We examine the linear stability of the base flow given by Eqs. (5)-(7) using a normal modes analysis. We decompose the flow variables and the concentration into steady base state quantities and two-dimensional, linear perturbations:

$$
(\tilde{u}, \tilde{v}, \tilde{p}, \tilde{c}, \tilde{\mu})(\tilde{x}, \tilde{y}, \tilde{t})=\left(U(\tilde{y}), 0, P, C(\tilde{y}), \mu_{0}(\tilde{y})\right)+(\hat{u}, \hat{v}, \hat{p}, \hat{c}, \hat{\mu})(y) \exp (i[\alpha \tilde{x}-\omega \tilde{t}])
$$


where the hat decoration designates the perturbation quantities. In Eq. (9), $\mu_{0}=e^{C \ln m}$ and $\hat{\mu}=\ln m e^{C \ln m} \hat{c}$ [53] represent the base state and perturbation viscosity, respectively; $\alpha$ is the disturbance wavenumber and $\omega$ its frequency, both of which can be complex. The amplitude of the velocity disturbances are then re-expressed in terms of a streamfunction: $(\hat{u}, \hat{v})=\left(\psi^{\prime},-\mathrm{i} \alpha \psi\right)$ where the prime denotes differentiation with respect to $y$. Substitution of Eq. (9) into the governing equations, Eqs. (2)-(4), subtraction of the base state equations, subsequent linearization and elimination of the pressure perturbation yields the following coupled ordinary differential eigenvalue equations (following the suppression of the hat decoration)

$$
\begin{gathered}
\mathrm{i} \alpha \operatorname{Re}\left[\left(\psi^{\prime \prime}-\alpha^{2} \psi\right)(\mathrm{U}-\omega / \alpha)-\mathrm{U}^{\prime \prime} \psi\right]=\mu_{0}\left(\psi^{\mathrm{iv}}-2 \alpha^{2} \psi^{\prime \prime}+\alpha^{4} \psi\right)+2 \mu_{0}^{\prime}\left(\psi^{\prime \prime \prime}-\alpha^{2} \psi^{\prime}\right)+ \\
\mu_{0}^{\prime \prime}\left(\psi^{\prime \prime}+\alpha^{2} \psi\right)+U^{\prime}\left(\mu^{\prime \prime}+\alpha^{2} \mu\right)+2 U^{\prime \prime} \mu^{\prime}+U^{\prime \prime \prime} \mu \\
\operatorname{i} \alpha \operatorname{Pe}\left[(\mathrm{U}-\omega / \alpha) \mathrm{c}-\psi \mu_{0}^{\prime}\right]=\left(\mathrm{c}^{\prime \prime}-\alpha^{2} \mathrm{c}\right) ;
\end{gathered}
$$

here, $\omega$ is the eigenvalue. Eq. (10) is an Orr-Sommerfeld equation, which is coupled to Eq. (11) through the dependence of the viscosity on the concentration. Equations (10) and (11) are solved subject to appropriate boundary conditions, which will be discussed below. The existence of the eigenfunctions $(\psi, c)(y ; \alpha)$ is contingent upon $\alpha$ and $\omega$ satisfying a dispersion relation, $D(\alpha, \omega ; m, \mathrm{Re}, \mathrm{Pe})$. In cases, wherein $\omega$ is complex (real) and $\alpha$ is real (complex), the modes are temporal (spatial) and $\omega=\omega(\alpha ; m, \operatorname{Re}, \mathrm{Pe})(\alpha=\alpha(\omega ; m, \operatorname{Re}, \mathrm{Pe}))$.

In order to determine whether the flow is stable or unstable, and, in the latter case, whether absolutely or convectively unstable, we follow an approach, which has been used previously to analyze the stability of mixing layers, jets and wakes, and in plasma flows [44, 54-58]. This is outlined in appendix A. To obtain numerical solutions of Eqs. (10) and (11), only half of the channel is considered, $y \in[1 / 2,1]$. This domain is decomposed into three regions, $1 / 2 \leq y \leq 1 / 2+h, 1 / 2+h \leq y \leq 1 / 2+h+q$ and $1 / 2+h+q \leq y \leq 1$, and the eigenfunctions in each region are then expanded using Chebyshev polynomials through a spectral method [53, 59, 60]. The decomposition of the domain endows the edges of the mixed layer with more points than its interior, thereby enhancing the resolution of the numerical solutions where the base state concentration and its derivatives must be continuous [53]. This is due to the distribution of Gauss-Lobatto points in the Chebyshev polynomials. 
Solutions are obtained subject to the following boundary conditions at the upper wall

$$
\psi=\psi^{\prime}=c=0 \quad \text { at } \quad y=1
$$

and either $\psi^{\prime}=\psi^{\prime \prime \prime}=c^{\prime}=0$ or $\psi=\psi^{\prime \prime}=c=0$ at $y=1 / 2$. The latter conditions are appropriate for 'sinuous' and 'varicose' modes; periodic conditions are employed in the $x$ direction. Our results (not shown) reveal that the sinuous mode is dominant for the range of parameters considered in the present work. Consequently, all of the results presented below correspond to sinuous modes exclusively.

\section{B. Results}

We begin the presentation of our results by demonstrating their convergence upon refinement of the spatial-mesh. Evidence of this is provided in Fig. 2, in which we plot $\omega_{i}$ against $\alpha_{r}$ for $\operatorname{Re}=500, \mathrm{Sc}=10, m=2, h=0.3$ and $q=0.05$; in this case, the linear, temporal stability of the system is considered. It can be seen that the curves are indistinguishable for different values of the order of Chebyshev polynomials, $N$. Thus, $N=121$ is used to generate the rest of the stability results in this paper. Also, inspection of Fig. 2 reveals the presence of a band of $\alpha_{r}$ for which $\omega_{i}>0$ and, therefore, of a linear, temporal instability, with well-defined high- and low-wavenumber 'cut-off' modes; the latter mode is associated with finite wavelength disturbances, rather than $\alpha_{r}=0$. We proceed below with a discussion of convective and absolute instabilities.

As discussed in section III A, for a given set of parameters, the value of $\omega_{0}$ corresponds to a pinch point singularity in the complex $\alpha$-plane. The process of identifying $\omega_{0}$ is illustrated in Fig. 3 and the isocontours of $\omega_{r}$ and $\omega_{i}$ for $m=25$ are shown in Figs. 3(a) and (b), respectively. Similarly, the isocontours of $\omega_{r}$ and $\omega_{i}$ for $m=40$ are given in Figs. 3(c) and (d), respectively; the rest of the parameters used to generate Fig. 3 are $\operatorname{Re}=500$, $\mathrm{Sc}=50, h=0.3$ and $q=0.05$. It can be seen that the values of $\omega$ for $m=25$ and $m=40$ are $2.966-0.076 \mathrm{i}$ and $2.112+0.045 \mathrm{i}$, respectively, indicating that the flow is convectively unstable for $m=25$ and absolutely unstable for $m=40$. The mapping from the complex $\omega$ plane to the complex $\alpha$ plane through the dispersion relation, performed using the Briggs method [54] outlined above, is shown in Fig. 4. The simultaneous occurrence of a pinch point in the $\alpha$-plane and a branch point in $\omega$-plane can seen Fig. 4. 
Adopting a similar procedure to that employed to generate Fig. 4, the boundary in $m$ Re space delineating the presence of convective and absolute instabilities is calculated and shown in Fig. 5. The base flow is absolutely unstable for the parameters above the curves in Fig. 5. The slope of the curves decreases sharply with increasing Re reaching a minimum, $\mathrm{Re}_{\mathrm{m}}$, before increasing again at relatively high Re. Thus, absolute instabilities appear to be present in this system over an intermediate range of Re values. For relatively low Re, a large degree of viscosity stratification is required for absolute instability, whereas increasing the level of inertial contribution renders the flow more convective for sufficiently large Re.

The effects of Sc and $h$ on the boundary of convective and absolute instability have also been investigated and are shown in Figs. 5(a) and (b), respectively. It can be seen in Fig. 5 (a) that decreasing the relative significance of diffusion through an increase in Sc increases the range of parameters over which the flow is absolutely unstable. The overall shape of the boundary curve remains unaltered with $\mathrm{Re}_{\mathrm{m}}$ shifting towards low Re values. We have found that increasing Sc has a similar effect to that of decreasing $q$ (not shown), as expected since decreasing diffusion contributions is reflected by thinner mixed regions.

In Fig. 5(b), it can be seen that decreasing the flow rate ratio of the less viscous fluid by lowering the value of $h$ also widens the absolutely unstable range in $m$-Re space and lowers $\mathrm{Re}_{\mathrm{m}}$. The effect of $h$ on the temporal stability of the system has been studied extensively in previous work $[50,53]$ that examined the influence of $h$ on the neutral stability curves. This work found the flow to be unstable at relatively low Re (approximately equal to 30 where the velocity at the channel centerline and the half-channel width were used as velocity and length scales, respectively), $S c=10^{5}$ and $m=1.05$, when the mixed layer overlaps with the critical layer, where the phase and base flow velocities are equal. In order to explore whether this mechanism is operative in our case, we have plotted the variation of $\omega_{i}$ with $\alpha_{r}$ for $m=2$ and $m=40$ in Figs. 6(a) and (b), respectively; these cases were chosen to represent convectively and absolutely unstable flows. The location of the critical layer corresponding to different $h$ for $m=2$ and $m=10$ is also given in table I. It can be seen that, for $m=2, h$ has a non-monnotonic influence on the growth rate of the most dangerous mode, which is maximized for an intermediate value of $h, h=0.25$. For this case, the critical layer lies approximately at the mid-point of the mixed layer. For $m=40$, however, increasing $h$ has a monotonically stabilizing influence, as shown in Fig. 6(b), despite the fact that the critical layer is located in the mixed layer for $h=0.2$ and $h=0.35$. 
In order to gain further insight into the mechanisms underlying the instabilities discussed in the foregoing, we have carried out an analysis of the 'energy' budget [16]; a similar analysis was also performed recently by Sahu et al. [59] and Sevlam et al. [51] for immiscible nonNewtonian channel flows and miscible core annular flows, respectively. The 'energy' equation is readily derived by taking the inner product of the vertical and horizontal components of the momentum conservation equation, Eq. (3), with their respective velocity components. The resultant equation is then averaged over the wavelength, $\lambda=2 \pi / \alpha_{r}$, and integrated over the channel height. Use of the Gauss-divergence theorem finally yields

$$
K I N=D I S+R E Y+A+B+F
$$

where the various terms appearing in Eq. (13) are given by

$$
\begin{gathered}
K I N \equiv \frac{1}{\lambda} \frac{\mathrm{d}}{\mathrm{d} t} \int_{0}^{1} \mathrm{~d} y \int_{0}^{\lambda} \mathrm{d} x\left[\frac{1}{2}\left(\hat{u}^{2}+\hat{v}^{2}\right)\right], \\
D I S \equiv-\frac{1}{\lambda \operatorname{Re}} \int_{0}^{1} d y \int_{0}^{\lambda} \mu_{0} \mathrm{~d} x\left[2\left(\frac{\partial \hat{u}}{\partial x}\right)^{2}+\left(\frac{\partial \hat{u}}{\partial y}+\frac{\partial \hat{v}}{\partial x}\right)^{2}+2\left(\frac{\partial \hat{v}}{\partial y}\right)^{2}\right], \\
R E Y \equiv \frac{1}{\lambda} \int_{0}^{1} \mathrm{~d} y \int_{0}^{\lambda} \mathrm{d} x\left[-\hat{u} \hat{v} \frac{\mathrm{d} U}{\mathrm{~d} y}\right], \\
A \equiv \frac{1}{\lambda} \int_{0}^{1} \mathrm{~d} y \int_{0}^{\lambda} \mathrm{d} x\left[\frac{d \mu_{0}}{d y} \frac{\partial \hat{v}^{2}}{\partial y}\right], \\
B_{x}+B_{y}=\frac{1}{\lambda \operatorname{Re}} \int_{0}^{1} d y \int_{0}^{\lambda} \mathrm{d} x\left[\hat{v} \frac{\partial \hat{\mu}}{\partial x} \frac{\mathrm{d} U}{d y}\right]+\frac{1}{\lambda \operatorname{Re}} \int_{0}^{1} d y \int_{0}^{\lambda} \mathrm{d} x\left[\hat{u} \frac{\partial \hat{\mu}}{\partial y} \frac{\mathrm{d} U}{d y}\right], \\
F \equiv-\frac{1}{\lambda \operatorname{Re}} \int_{0}^{1} d y \int_{0}^{\lambda} \mathrm{d} x\left[\hat{u} \hat{\mu} \frac{\mathrm{d}^{2} U}{\mathrm{~d} y^{2}}\right] .
\end{gathered}
$$

Here, $K I N$ represents the temporal rate of change of the disturbance kinetic energy: $K I N>$ 0 indicates the presence of instability; $R E Y$ denotes the 'Reynolds stress' term, which determines the rate of transfer of energy from the base flow to the disturbances, and DIS corresponds to the viscous dissipation of energy. The term $A$ represents the energy of the disturbances due to mean viscosity gradients, while the disturbance energies $B$ and $F$ are due to the gradient of viscosity perturbations and viscosity perturbations, respectively. The term $B$ can be further decomposed into $B_{x}$ and $B_{y}$, where $B_{x}$ and $B_{y}$ are the disturbance energies associated with the gradient of viscosity perturbations in the $x$ and $y$ directions, respectively. 
In Figs. 7(a) and (b), we present a breakdown of $K I N$ into its constituent components as a function of Re for $m=30$ and $\mathrm{Sc}=100$, and $m=40$ and $\mathrm{Sc}=50$, respectively; the rest of the parameter values are $h=0.3$ and $q=0.05$. The wavenumber chosen to generate the results in Fig. 7 corresponds to that associated with the maximal temporal growth rate at each Re considered. Inspection of Fig. 7 reveals that the largest contributor to instability is $B_{y}$, which is the energy associated with the gradient of viscosity perturbations in the $y$ direction. Sevlam et al. [51] also reached a similar conclusion for miscible core-annular flows at high Schmidt numbers. This magnitude of $B_{y}$ decreases with increasing Re in contrast to that of $R E Y$, which makes a positive, albeit small, contribution to $K I N$. The disturbance energy associated with mean viscosity gradients and perturbation viscosity gradients in the $x$-direction, represented by $A$ and $B_{x}$, respectively, is small for all parameter values considered. Viscous dissipation and disturbance energy associated with viscosity perturbations, represented by $D I S$ and $F$, respectively, are stabilizing although their magnitude decreases with Re. In the following section, the stability of the flow is probed in the nonlinear regime and connections with the results presented in this section will be highlighted wherever possible.

\section{DIRECT NUMERICAL SIMULATIONS}

In this section, we present the results of direct numerical simulations of the flow via solution of Eqs. (2)-(4) over a wide range of parameters.

\section{A. Numerical procedure}

In this section, we describe the methodology used to solve Eqs. (2)-(4) in order to simulate two-fluid miscible channel flow. A staggered grid is used for the finite-volume discretization of these equations, with the scalar variables, the pressure and concentration, defined at the center of each cell while the velocity components are defined at the cell faces.

The solutions of the governing equations are coupled in the following manner: we first update the concentration field by solving Eq. (4) with the velocity field at time steps $n$ and $n-1$; this field is then updated to time-step $n+1$ via solution of Eq. (3) in conjunction with the continuity equation, Eq. (2). For the spatial discretization, the advective 
term, the second term on the left-hand-side of Eq. (4), is approximated using a weighted essentially non-oscillatory (WENO) scheme; central difference schemes are used to discretize the diffusion term on the right-hand-side of Eq. (4). For the temporal discretization, the Adams-Bashforth and Crank-Nicolson methods are used for the advective and second-order dissipation terms, respectively, in order to achieve second-order accuracy. The numerical procedure described here was previously employed by Ding et al. [61] to solve Eqs. (2) and (3) along with a Cahn-Hilliard equation for the for the position of the interface within the framework of the "diffuse interface" method. This procedure has been validated by simulating Rayleigh-Taylor instabilities in incompressible two-phase flows with density contrasts [61] and droplet spreading $[62,63]$, and was applied to physical problems such as droplet deformation due to shear flow [64] in the presence of moving contact lines.

The predictions of our numerical procedure are validated against those obtained from a linear (temporal) stability analysis for the case shown in Fig. 2. The comparison between the numerical solutions of Eqs. (2)-(4) and the linear theory predictions for $\alpha_{r}=4$, corresponding to the most dangerous linear mode, are depicted in Fig. 8. Here, the latter are represented by the maximal vertical velocity component, $v_{\max }$, which is equivalent to $\hat{v} e^{\mathrm{i}\left(\alpha_{r} x-\omega t\right)}$; the former are obtained over a spatial domain of length $2 \pi / \alpha_{r}$ by starting from the eigenfunctions corresponding to $\alpha_{r}=4$ as an initial condition with amplitude equal to $1 \times 10^{-3}$. Periodic boundary conditions are imposed in the $x$-direction. The numerical simulation in Fig. 8 is carried out for 256 points in each direction. We have checked this result for different sets of grid points and found convergence for grid points greater than or equal to 161 in both the $x$ and $y$ directions. Inspection of Fig. 8(a) reveals excellent agreement for sufficiently early times prior to the onset of nonlinearities. Figures 8(b) and (c) also show a comparison in terms of contour plots of $v_{\max }$ obtained from the linear stability analysis and the full numerical simulations, for the same parameter values as those used to generate Fig. 8(a) at $t=1$ and $t=6$ respectively. Excellent agreement is found in the comparison of the contour plots of $v_{\max }$ at early times, but at later times, the contours of $v_{\max }$ diverge due to the onset of nonlinearities. These results inspire confidence in the predictions of the numerical procedure used to solve Eqs. (2)-(4); similar agreement was also obtained in terms of the concentration (not shown). We have also carried out mesh refinement tests for the extended domain case, as will be discussed in the next section, and the results below were obtained using 81 and 1001 grid points in the $x$ and $y$-directions, respectively, for channels 
of aspect ratio up to $1: 40$.

\section{B. Three-layer flow}

Here, we discuss the results of our numerical simulation of the three-layer flow configuration, shown schematically in Fig. 1; the objective of this work is to establish a connection between the results shown in Figs. 3-5 and those obtained in the nonlinear regime. In Figs. 9(a,c) and 10, and Figs. 9(b,d) and 12, we show numerical solutions for $m=2$ and $m=30$, respectively, and the rest of the parameter values are $\mathrm{Re}=500, \mathrm{Sc}=100$ and $h=0.3$; $q$ is set to 0.05 initially. As suggested by Fig. 5(a), one would expect the flow associated with these parameters to exhibit convective and absolute instabilities, respectively. Periodic oscillations of $h$ with amplitude equal to $10^{-3}$ are applied at the inlet; this is referred to below as inlet 'forcing'. The oscillation frequency corresponds to that at the branch point singularity obtained by following the procedure outlined in section III A (cf. Fig. 4); these frequencies are equal to 4.03 and 2.556 for $m=2$ and $m=30$, respectively.

Figures 9 (a) and (b) show the contours of $c=0.5$ at different times for $m=2$ and $m=30$, respectively, at $t=4,12,20$. It is clearly seen that, whereas for $m=2$ the amplitude of the disturbances near the inlet remains small and grows downstream, as expected from a flow dominated by a convective instability, the $m=30$ case is characterized by disturbance growth near the inlet region; this reflects the presence of an absolute instability, in agreement with the predictions shown in Fig. 5(a). In Figs. 9(c) and (d), we show enlarged views of the $c=0.5$ contour near the inlet region, which provide estimates for the disturbance wavelength in that region for $m=2$ and $m=30$, respectively. It is seen that the value of these estimates are 1.4 and 0.56 , respectively, which are approximately equal to the wavelength of the most dangerous temporal mode for $m=2$ and that at the saddle point corresponding to the pinch point singularity for $m=30$ (see Figs. 9(e) and (f)). Thus, the periodic forcing at the inlet drives the flow with temporal linear dynamics and 'saddle point dynamics' in the convectively and absolutely unstable cases, respectively.

Concentration contours are shown in Figs. 10 and 12 for $m=2$ and $m=30$ with the rest of the parameters remaining unchanged from Fig. 9. In Fig. 10, it is seen that the disturbances, which originate near the inlet grow in amplitude as they are convected downstream. These then lead to 'roll-up' phenomena that bring about the formation of 
large vortical structures, shown in Fig. 11, which depicts contours of the vorticity, and the entrainment of the more viscous fluid, fluid ' 1 ', near the wall into the channel core. The large vortices are, in turn, also convected downstream leaving behind a three-layer structure characterized by a relatively smooth inlet region, small-amplitude vortices half-way along the channel, followed by a seemingly stable region near the channel outlet; in this region, mixing of the two fluids occurs by diffusive processes. The analogous results associated with $m=30$, shown in Fig. 12, exhibit somewhat similar dynamics to those depicted in Fig. 10. The 'roll-up' phenomena for $m=30$ gives rise to vortical patterns that persist to later times than for $m=2$, as expected from a case dominated by an absolute instability. It should be noted that the thickness of the mixed layer increases downstream from the inlet due to diffusion, which leads to spatially-varying $h$ and $q$ values; this naturally complicates comparisons with the predictions presented in Fig. 5 based on constant $h$ and $q$.

It is instructive to compare the flow dynamics in the presence and absence of inlet forcing. The effect of forcing is shown in Fig. 13, which depicts concentration contours for $m=2$ and $m=30$ in panels (a) and (c), and (b) and (d), respectively; the rest of the parameters remain unaltered from Figs. 10-12. Inspection of Fig. 13 reveals that, in the $m=2$ case, which, based on Fig. 5 is convectively unstable, the absence of inlet forcing leads to a relatively stable flow devoid of vortical patterns. In contrast, the dynamics in the $m=30$ case appear to be very weakly-dependent on the presence of forcing, as might be expected from an absolutely unstable situation. The remainder of the results discussed in this subsection were generated with inlet forcing.

The influence of Sc and Re on the dynamics of the three-layer configuration was also investigated. In Fig. 14, we show the effect of decreasing the value of Sc from 100 to 50, thereby rendering the flow more diffusive, while keeping the rest of the parameters unaltered from Fig. 12. According to the results shown in Fig. 5(a), one would expect the flow in this case to exhibit convective instabilities. This is indeed reflected by the spatio-temporal evolution of the concentration contours shown in Fig. 14, which reveals the development of 'roll-up' patterns that are convected rapidly downstream. As can also be seen in Fig. 14, vortical patterns develop again at relatively late times following a relatively quiescent period between $t=30-45$. We have ensured that this intermittent behaviour is not a spurious numerical artefact associated, for instance, with an insufficient domain length: increasing the channel length by $50 \%$ yielded identical results to those shown in Fig. 14. Variation of 
Re also has a profound effect on the flow characteristics. Comparison of Figs. 15 and 16 in which $\operatorname{Re}=100$ and $\operatorname{Re}=1000$, respectively, demonstrates that a tenfold increase in $\operatorname{Re}$ gives rise to significant destabilization of the flow. This is characterized by the relatively rapid development of large vortices over a substantial proportion of the channel, leading to significant entrainment of the more viscous fluid ' 1 ' into the channel core, as is also shown in Fig. 16. This, in turn, leads to the development of two distinct regions: one spanning the spatial interval between the inlet and the channel mid-point, characterized by a thin layer of fluid ' 1 ' adjacent to the walls of almost uniform thickness; the other region extends from the mid-point to the channel exit having a considerable thinner, wavy viscous layer. The results generated for the displacement flows are presented next.

\section{Displacement flow}

In this section, we describe the results obtained for the case wherein a fluid initially occupying the channel completely is displaced by a less viscous one. In Fig. 18, we show the spatio-temporal evolution of the concentration contours for $\mathrm{Re}=500, \mathrm{Sc}=20$ and $m=10$; the channel aspect ratio is $1: 40$. As can be seen from this figure, a 'finger' of fluid ' 2 ' penetrates the more viscous fluid ' 1 ' at early times. As the finger length increases, instabilities develop rendering the 'interface' between the two fluids wavy, leading to the formation of vortical patterns that destroy the integrity of the finger; this is accompanied by intense, convective mixing of the two fluids. The region from the channel inlet to its midpoint resembles that associated with the three-layer flow structure discussed in section IV B above as the penetrating finger of fluid ' 2 ' leaves behind a thin layer of the more viscous fluid adjacent to the wall. Downstream of the channel mid-point, the three-layer flow comprises a very thin, viscous wall layer and a slender, wavy finger of the less viscous fluid, separated by thick mixed regions.

In Fig. 19(a), we plot the temporal evolution of a dimensionless measure of the mass of fluid ' 1 ' left in the channel, $M_{0.95} / M_{0}$; here, $M_{0.95}$ and $M_{0}$ represent, respectively, the mass of fluid with $c \geq 0.95$ and that of fluid ' 1 ' occupying the channel initially. In Fig. 19(b), we show the variation with time of the spatial location of the leading front separating the two

fluids, $x_{\text {tip }}$. Inspection of Fig. 19(a) reveals that the mass fraction of the displaced, more viscous fluid decreases from unity, essentially linearly at relatively early times (viz. the line 
of constant slope in this panel), following the penetration of the less viscous, largely stable finger, as shown in the top two panels of Fig. 18. The rate of decrease of the mass fraction, corresponding to the displacement rate of the more viscous fluid, is accelerated following the onset and development of the instabilities discussed above, shown in the third and fourth panels of Fig. 18, which act to mix the fluids efficiently; this occurs between $t \approx 10-25$. Following this period of time, the displacement rate decreases since the flow, at this relatively late stage of the dynamics, is dominated by diffusion that acts to mix the fluids on longer time scales. Thus, Fig. 19(a) indicates the existence of three flow regimes: unperturbed penetration of the less viscous into the more viscous one at relatively early times; followed by an instability-dominated regime with convective mixing and acceleration of the displacement rate at intermediate times; this, in turn, is followed by a diffusion-dominated regime and deceleration of the displacement rate at late times. Despite the complex dynamics shown in Fig. 18(a) giving rise to displacement rates observed in Fig. 19(a), the slope of $x_{\text {tip }}$ with time remains remarkably linear, indicating that the leading front propagates with an approximately constant speed, $u_{\text {tip }}$. Inspection of Fig. 19 also reveals that convergence of the results has indeed been achieved upon mesh refinement. The results discussed in the rest of this paper were generated using $41 \times 701$ grid points.

We have also carried out a parametric study to investigate the effect of varying Re, Sc and $m$ on the displacement characteristics. In Figs. 20-22, it is seen that increasing the value of Re from 100, to 500 and then 1000, respectively, leads to the rapid development of instabilities that lead to complex dynamics and intricate flow patterns. These are punctuated by more pronounced 'roll-up' phenomena and intense mixing that lead to an increase in the rates of displacement and a decrease in the duration of the diffusive mixing period, as shown in Figs. 28(a) and (b). In contrast, at the relatively low value of $R e=100$, the penetrating finger of the less viscous fluid propagates in an apparently stable manner, and fluid mixing is dominated by diffusive processes on the time scales shown in Fig. 20. The rest of the parameter values in Figs. 20-22 are $\mathrm{Sc}=100$ and $m=30$, which, according to the results presented in Fig. 5, may suggest that the flow should exhibit absolute and convective instabilities for $\operatorname{Re}=500$, and $\operatorname{Re}=100$ and $\mathrm{Re}=1000$, respectively. However, it should be noted that one can only estimate the thickness of the more viscous wall layer left behind by the penetrating, less viscous finger that leads to the formation of the three-layer structure whose linear stability was investigated in section III. Such a structure is indeed established 
locally near the inlet region in Figs. 20-22, but the value of $h$ in this case varies, albeit weakly, with $x$, and $q$ also increases due to diffusion, thereby complicating any connections that may be established with the results of section III.

The effect of decreasing Sc is to promote diffusive mixing, manifested by the rapid smearing of the initially sharp boundaries between fluids ' 1 ' and ' 2 ' and the absence of instabilities, as shown in Fig. 23 for $\mathrm{Sc}=1, \mathrm{Re}=500$ and $m=25$. The displacement rate also appears to be approximately constant for the large majority of the flow, as depicted in Fig. 28(c). Increasing Sc to 10 and then 100 in Figs. 24 and 25, respectively, allows the magnitude of the vertical gradients in the viscosity perturbations to be maintained for longer times since diffusive effects are weaker at these larger Sc values. As was shown in section III B via an energy analysis, these gradients provide the largest positive contribution to the rate of change of the disturbance kinetic energy and are therefore highly destabilizing. As shown in Figs. 24 and 25, larger Sc flows are dominated by instabilities, complex dynamics and convective mixing, which, interestingly, appear to prolong the displacement of the more viscous fluid from the channel (see Fig. 28(c)), although the displacement rates saturate at sufficiently large Sc. Inspection of Fig. 28(d) also shows that the speed of propagation of the leading front, $u_{\text {tip }}$, is essentially insensitive to variations in Sc.

Finally, we examine the effect of varying $m$ on the displacement dynamics. As shown in Fig. 26, generated with $m=2, \operatorname{Re}=500$ and $\mathrm{Sc}=100$, the penetrating finger of fluid ' 1 ', which occupies a large fraction of the channel width at early times, develops a sharp 'nose' at later times (see the fourth panel of Fig. 26 corresponding to $t=20$ ). Instabilities set in at relatively late times due to the relative weakness of the viscosity stratification in this case. This is in contrast to the flow characterized by $m=40$ case shown in Fig. 27, which is accompanied by the development of 'roll-up' and vigorous convective mixing of the fluids; this also leads to the acceleration of the displacement of the more viscous fluid from the channel, as shown in Figs. 28(e) and (f).

\section{CONCLUDING REMARKS}

We have investigated the stability of miscible two-fluid flow in a horizontal channel using linear stability theory and direct numerical simulations. The equations governing this flow are the continuity and Navier-Stokes equations in which the viscosity is a function of the 
concentration of the more viscous fluid; the dynamics of this concentration are governed by a convective-diffusion equation. This system of equations is parameterized by a Reynolds number, Re, a Schmidt number, Sc and a viscosity ratio, $m$.

In order to determine the linear stability characteristics of the flow, we have derived an Orr-Sommerfeld equation for the disturbance streamfunction coupled to a linearized convective-diffusion equation for the disturbance concentration. These are ordinary differential eigenvalue equations wherein the complex growth rate is the eigenvalue; the wavenumber in this equation is taken to be complex. A Briggs-type analysis was then carried out to delineate the boundaries between linear convective and absolute instability in $m-$ Re space as a parametric function of Sc. This was performed for a three-layer structure comprising layers of the more viscous fluid adjacent to the wall and the less viscous fluid in the channel core; the fluids are separated by a mixed layer. This analysis showed that increasing Sc widens the range of parameters over which the flow is absolutely unstable. An energy analysis also revealed that the vertical gradients in the viscosity perturbations are primarily responsible for instability in this flow, followed by the Reynolds stress terms, which are also destabilizing. Our transient numerical simulations allowed us to probe the flow stability in the nonlinear regime and revealed the development of 'roll-up' phenomena, cellular patterns and convective mixing for sufficiently large Re, Sc and $m$ values.

\section{Acknowledgments}

The authors acknowledge fruitful discussions with Dr. P. D. M. Spelt. They also thank the EPSRC for their support through grant numbers EP/E046029/1 and EP/D503051 and the DTI through grant number TP//ZEE/6/1/21191.

\section{APPENDIX A: METHOD FOR DETECTING ABSOLUTE AND CONVECTIVE INSTABILITIES}

The approach employed to distinguish between absolute and convective instabilities involves the association of a differential operator for the perturbations ( $\psi$ and $c$ in the present case) in physical space with the dispersion relation in complex $(\omega, \alpha)$ space and the subsequent introduction of a Green's function, $G(x, y, t)$, that represents the impulse response of 
the flow. The latter can be expressed as a double Fourier integral in the complex $\omega$ and $\alpha$ planes along contours, $C_{\omega}$ and $C_{\alpha}$ respectively, which are chosen in order to satisfy causality considerations. Variation of $\omega$ along $C_{\omega}$ is associated with spatial branches in the complex $\alpha$ plane, $\alpha^{+}$and $\alpha^{-}$, located in the upper and lower half planes, respectively. The contour $C_{\alpha}$ lies between $\alpha^{+}$and $\alpha^{-}$in order to satisfy causality requirements.

In order to determine the stability characteristics, the behaviour of the $G(x, y, t)$ at long times is determined along different 'rays' corresponding to constant values of $x / t$. This is done by first using the method of steepest descent [65], which involves determining the saddle point of the exponent of $G, \alpha_{*}$. The saddle point condition is

$$
\frac{\partial \omega}{\partial \alpha}\left(\alpha_{*}\right)=\frac{x}{t}
$$

which is real and corresponds to the group velocity. The contour $C_{\alpha}$, originally coinciding with the $\alpha_{r}$ axis, is then deformed into the path of steepest descent through $\alpha_{*}$, so that the behaviour of $G(x, y, t)$ is dominated by the contribution of the region nearest $\alpha_{*}$ :

$$
G(x, y, t) \sim \frac{e^{i\left[\pi / 4+\alpha_{*} x-\omega\left(\alpha_{*}\right) t\right]}}{\frac{\partial D}{\partial \omega}\left[\alpha_{*}, \omega\left(\alpha_{*}\right)\right]\left[2 \pi \frac{\partial^{2} \omega}{\partial \alpha^{2}}\right]^{1 / 2}} .
$$

As can be seen from Eq. (A2), the temporal growth rate measured by an observer moving along a ray $x / t$ is $\omega_{*, i}-(x / t) \alpha_{*}$. To determine whether the flow is linearly stable or unstable, one first finds the maximum temporal growth rate, $\omega_{i, \max }=\omega_{i}\left(\alpha_{\max }\right)$ where $\alpha_{\max }$ is real and satisfies $\partial \omega_{i} / \partial \alpha\left(\alpha_{\max }\right)=0$. The flow is then said to be

- linearly stable if $\omega_{i, \max }<0$ and

- linearly unstable if $\omega_{i, \max }>0$.

In order to determine whether the flow is convectively or absolutely unstable, one first determines the so-called "absolute frequency", $\omega_{0}=\omega\left(\alpha_{0}\right)$, where $\alpha_{0}$, the "absolute wavenumber", may be complex and satisfies

$$
\frac{\partial \omega}{\partial \alpha}\left(\alpha_{0}\right)=0
$$

this corresponds to the ray $x / t=0$ or zero group velocity. The "absolute growth rate", $\omega_{0, i}$, measures disturbance growth or decay along the $x / t=0$ ray, i.e. in a stationary reference frame. The flow is then said to be $[54,55]$ 
- convectively unstable if $\omega_{0, i}<0$ and

- absolutely unstable if $\omega_{0, i}>0$.

Physically, if a localized disturbance generated by an impulse spreads both upstream and downstream from its source, the flow is absolutely unstable; if, on the other hand, the disturbance amplitude grows downstream of the source, the flow is convectively unstable.

The $x / t=0$ condition given by Eq. (A3) coincides with the contour $C_{\omega}$ coming into contact with the locus of any temporal branches in the complex $\omega$ plane at $\omega_{0}$; if this event is accompanied by $C_{\omega}$ being in the upper (lower) half planes then the flow is absolutely (convectively) unstable. The zero group velocity condition also coincides with the pinching of the contour $C_{\alpha}[54]$ when the branches $\alpha^{+}$and $\alpha^{-}$, initially located in the upper and lower half planes, respectively, come into contact at $\alpha_{0}$. Evidence for the presence of such singularities in both the complex $\alpha$ and $\omega$ planes will be presented below.

[1] D. D. Joseph, R. Bai, K. P. Chen, and Y. Y. Renardy, "Core-annular flows," Ann. Rev. Fluid Mech. 29, 65 (1997).

[2] Q. Cao, L. Ventresca, K. R. Sreenivas, and A. K. Prasad, "Instability due to viscosity stratification downstream of a centreline injector," Can. J. Chem. Eng. 81, 913 (2003).

[3] M. Regner, M. Henningsson, J. Wiklund, K. Östergren and C. Trägårdh, "Predicting the displacement of yoghurt by Water in a pipe using CFD," Chem. eng. Technology 30, 844 (2007).

[4] C. S. Yih, "Instability due to viscous stratification," J. Fluid Mech. 27, 337 (1967).

[5] C. E. Hickox, "Instability due to viscosity and density stratification in axisymmetric pipe flow," Phys. Fluids 14, 251 (1971).

[6] D. D. Joseph, M. Renardy, and Y. Y. Renardy, "Instability of the flow of two immiscible liquids with different viscosities in a pipe," J. Fluid Mech. 141, 309 (1984).

[7] H. H. Hu and D. D. Joseph, "Lubricated pipelining: stability of core-annular flows. Part 2," J. Fluid Mech. 205, 395 (1989).

[8] D. D. Joseph and Y. Y. Renardy, Fundamentals of Two-Fluid Dynamics. Part I: Mathemtical Theory and Applications (Springer-Verlag, New York, 1992). 
[9] C. Kouris and J. Tsamopoulos, "Dynamics of axisymmetric core-annular flow in a straight tube. I. The more viscous fluid in the core, bamboo waves," Phys. Fluids 13, 841 (2001).

[10] C. Kouris and J. Tsamopoulos, "Dynamics of axisymmetric core-annular flow in a straight tube. II. The less viscous fluid in the core, saw tooth waves," Phys. Fluids 14, 1011 (2002).

[11] M. K. Smith, "The mechanism for the long-wave instability in a thin liquid film," J. Fluid Mech. 217, 469 (2001).

[12] F. Charru and E. J. Hinch, "Phase diagram' of interfacial instabilities in a two-layer Couette flow and mechanism for the long-wave instability," J. Fluid Mech. 414, 195 (2000).

[13] A. P. Hooper and W. G. C. Boyd, "Shear flow instability at the interface between two fluids," J. Fluid Mech. 128, 507 (1983).

[14] E. J. Hinch, "A note on the mechanism of the instability at the interface between two shearing fluids," J. Fluid Mech. 144, 463 (1984).

[15] S. G. Yiantsios and B. G. Higgins, "Numerial solution of eigenvalue problems using the compound matrix-method," J. Comp. Phys. 74, 25 (1988).

[16] P. A. M. Boomkamp and R. H. M. Miesen, "Classification of instabilities in parallel two-phase flow," Int. J. Multiphase Flow 22, 67 (1996).

[17] Y. Y. Renardy and D. D. Joseph, "Couette-flow of two fluids between concentric cylinders," J. Fluid Mech. 150, 381 (1985).

[18] L. Preziosi, K. Chen, and D. D. Joseph, "Lubricated pipelining: stability of core-annular flow," J. Fluid Mech. 201, 323 (1989).

[19] H. H. Hu, T. S. Lundgren, and D. D. Joseph, "Stability of core-annular flow with a small viscosity ratio," Phys. Fluids A 2, 1945 (1990).

[20] H. H. Hu and N. Patankar, "Non-axisymmetric instability of core-annular flow," J. Fluid Mech. 290, 213 (1995).

[21] M. E. Charles, G. W. Govier, and G. W. Hodgson, "The horizontal pipeline flow of equal density oil-water mixtures," Can. J. Chem. Eng. 39, 27 (1961).

[22] R. Bai, K. Chen, and D. D. Jospeh, "Lubricated pipelining: stability of core annular flow. Part 5. Experiments and comparison with theory," J. Fluid Mech. 240, 97 (1992).

[23] J. Li and Y. Y. Renardy, "Direct simulation of unsteady axisymmetric core-annular flow with high viscosity ratio," J. Fluid Mech. 391, 123 (1999).

[24] C. Kouris and J. Tsamopoulos, "Core-annular flow in a periodically constricted circular tube. 
Part 1. Steady-state linear stability and energy analysis," J. Fluid Mech. 432, 31 (2001).

[25] C. Kouris and J. Tsamopoulos, "Core-annular flow in a periodically constricted circular tube. Part 2. Nonlinear dynamics," J. Fluid Mech. 470, 181 (2002).

[26] H. H. Wei and D. S. Rumschitzki, "The linear stability of a core-annular flow in an asymptotically corrugated tube," J. Fluid Mech. 466, 113 (2002).

[27] H. H. Wei and D. S. Rumschitzki, "The weakly nonlinear interfacial stability of a core-annular flow in a corrugated tube," J. Fluid Mech. 466, 149 (2002).

[28] B. T. Ranganathan and R. Govindarajan, "Stabilisation and destabilisation of channel flow by location of viscosity-stratified fluid layer," Phys. Fluids. 13(1), 1 (2001).

[29] R. Govindarajan, "Effect of miscibility on the linear instability of two-fluid channel flow," International J. Multiphase Flow 30, 1177 (2004).

[30] P. Ern, F. Charru, and P. Luchini, "Stability analysis of a shear flow with strongly stratified viscosity," J. Fluid Mech. 496, 295 (2003).

[31] G. I. Taylor, "Deposition of viscous fluid on the wall of a tube," J. Fluid Mech. 10, 161 (1961).

[32] B. G. Cox, "On driving a viscous fluid out of a tube," J. Fluid Mech. 14, 81 (1962).

[33] P. Petitjeans and T. Maxworthy, "Miscible displacements in capillary tubes. Part 1. Experiments," J. Fluid Mech. 326, 37 (1996).

[34] C.-Y. Chen and E. Meiburg, "Miscible displacement in capillary tubes. Part 2. Numerical simulations," J. Fluid Mech. 326, 57 (1996).

[35] J. Kuang, T. Maxworthy, and P. Petitjeans, "Miscible displacements between silicone oils in capillary tubes," Eur. J. Mech. 22, 271 (2003).

[36] R. Balasubramaniam, N. Rashidnia, T. Maxworthy, and J. Kuang, "Instability of miscible interfaces in a cylindrical tube," Phys. Fluids 17, 052103 (2005).

[37] E. Lajeunesse, J. Martin, N. Rakotomalala, and D. Salin, "3D instability of miscible displacements in a Hele-Shaw cell," Phys. Rev. Lett. 79, 5254 (1997).

[38] E. Lajeunesse, J. Martin, N. Rakotomalala, D. Salin, and Y. C. Yortsos, "Miscible displacement in a Hele-Shaw cell at high rates," J. Fluid Mech. 398, 299 (1999).

[39] J. Scoffoni, E. Lajeunesse, and G. M. Homsy, "Interface instabilities during displacement of two miscible fluids in a vertical pipe," Phys. Fluids 13, 553 (2001).

[40] C. Gabard and J.-P. Hulin, "Miscible displacement of non-Newtonian fluids in a verticl tube," Eur. Phys. J. E. 11, 231 (2003). 
[41] M. d'Olce, J. Martin, N. Rakotomalala, D. Salin, and L. Talon, "Pearl and mushroom instability patterns in two miscible fluids' core annular flows," Phys. Fluids 20, 024104 (2008).

[42] B. Selvam, S. Merk, R. Govindarajan, and E. Meiburg, "Stability of miscible core-annular flows with viscosity stratification," J. Fluid Mech. 592, 23 (2007).

[43] S. V. Malik and A. P. Hooper, "Linear stability and energy growth of viscosity stratified flows," Phys. Fluids 17, 024101 (2005).

[44] P. Huerre and P. A. Monkewitz, "Local and global instability in spatially developing flows," Ann. Rev. Fluid Mech. 22, 473 (1990).

[45] J.-M. Chomaz, "Global instabilities in spatially developing flows: non-normality and nonlinearity," Ann. Rev. Fluid Mech. 37, 357 (2005).

[46] P. J. Schmid and D. S. Henningson, Stability and Transition in Shear Flows (Springer, New York, 2001).

[47] R. Valette, P. Laure, Y. Demay, and j. F. Agassant, "Convective linear stability analysis of two-layer coextrusion flow for molten polymers," J. of Non-Newtonian Fluid Mechanics 121, 41 (2004).

[48] S. H. Vanaparthy, C. Barthe, and E. Meiburg, "Density-driven instabilities in capillary tubes: influence of a variable diffusion coefficient," Phys. Fluids 18, 048101 (2006).

[49] C. T. Tan and G. M. Homsy, "Stability of miscible displacements: rectangular flow," Phys. Fluid 29, 73549 (1986).

[50] R. Govindarajan, "Effect of miscibility on the linear instability of two-fluid channel flow," International J. Multiphase Flow 30, 1177 (2004).

[51] B. Selvam, S. Merk, R. Govindarajan, and E. Meiburg, "Stability of miscible core-annular flows with viscosity stratification,” J. Fluid Mech. 592, 23 (2007).

[52] R. Govindarajan, S. V. L'vov, and I. Procaccia, "Retardation of the onset of turbulence by minor viscosity contrasts," Phys. Rev. Lett. 87, 174501 (2001).

[53] T. Theofanous, S. Sushchikh, and R. Nourgaliev, "Linear stability of sharp and diffuse interfaces under shear," FEDSM07 $5^{\text {th }}$ joint ASME/JSME fluids engineering summer conference, San Diego, California, USA (2007).

[54] R. J. Briggs, Research monograph no. 29 (MIT Press, Cambridge, 1964).

[55] A. Bers, in Handbook of Plasma Physics, edited by M. N. Rosenbluth and R. Z. Sagdeev (PUBLISHER, Amsterdam: North Holland, 1983), Vol. 1, pp. 451-517. 
[56] I. Vihinen, A. M. Honohan, and S. P. Lin, "Image of absolute instability in a liquid jet," Phys. Fluid 9 (11), 3117 (1997).

[57] W. O. Criminale, T. L. Jackson, and R. D. Roslin, Theory and Compuation of Hydrodynamic Stability (Cambridge University Press, Cambridge, UK, 2003).

[58] L. Kaiktsis and P. A. Monkewitz, "Global destabilization of flow over a backward-facing step," Phys. Fluid 15 (12), 3647 (2003).

[59] K. C. Sahu, P. Valluri, P. D. M. Spelt, and O. K. Matar, "Linear instability of pressure-driven channel flow of a Newtonian and Herschel-Bulkley fluid," Phys. Fluids 19, 122101 (2007).

[60] P. A. M. Boomkamp, R. H. M. Miesen, and G. V. Beijnon, "A Chebyshev collocation method for solving two-phase flow stability problems," J. Comp. Phys. 132, 191 (1997).

[61] H. Ding, P. D. M. Spelt, and C. Shu, "Diffuse interface model for incompressible two-phase flows with large density ratios," J. Computational Physics 226, 2078 (2007).

[62] H. Ding and P. D. M. Spelt, "Wetting condition in diffuse interface simulations of contact line motion," Phys. Rev. E 75, 046708 (2007).

[63] H. Ding and P. D. M. Spelt, "Inertial effects in droplet spreading: a comparison between diffuse-interface and level-set simulations," J. Fluid Mech. 576, 287 (2007).

[64] H. Ding and P. D. M. Spelt, "Onset of motion of three-dimensional droplet on a wall in a shear flow at moderate Reynolds numbers," J. Fluid Mech. 599, 341 (2008).

[65] C. M. Bender and S. A. Orszag, Advanced Mathematical Methods for Scientists and Engineers: Asymptotic Methods and Perturbation Theory (McGraw-Hill, New York, 1978). 
TABLE I: Variation of the critical layer location with $h$ for $\mathrm{Re}=500$ and $\mathrm{Sc}=50$. Two different values of $m$ are considered, $m=2$ and $m=40$, and the width of the mixed layer is $q=0.05$.

\begin{tabular}{|c|c|c|}
\hline$h$ & Location of critical layer $(m=2)$ & Location of critical layer $(m=40)$ \\
\hline 0.2 & 0.201 & 0.205 \\
\hline 0.25 & 0.270 & 0.238 \\
\hline 0.3 & 0.295 & 0.298 \\
\hline 0.35 & 0.343 & 0.353 \\
\hline
\end{tabular}




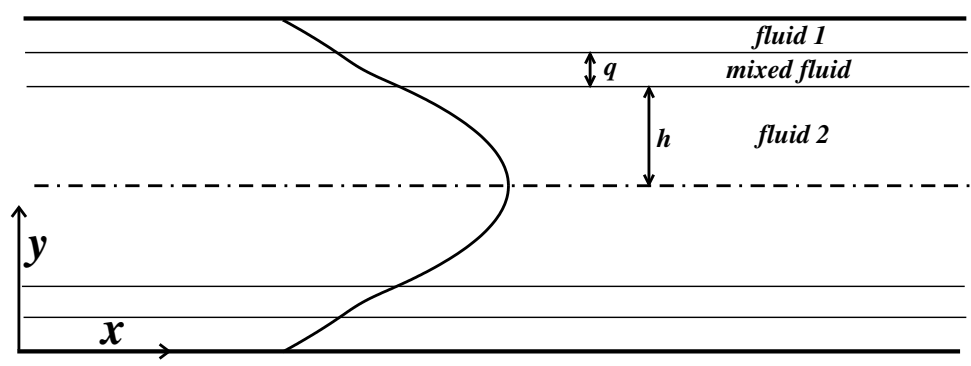

FIG. 1: Schematic of the 'three-layer' base state flow whose linear stability is analysed. Here, layers of fluids ' 1 ' and '2', corresponding to the more and less viscous fluids, respectively, are separated by mixed regions of thickness, $q$. The interfaces between these regions and the region occupied by the less viscous fluid are located at $y=1 / 2 \pm h$, respectively. 


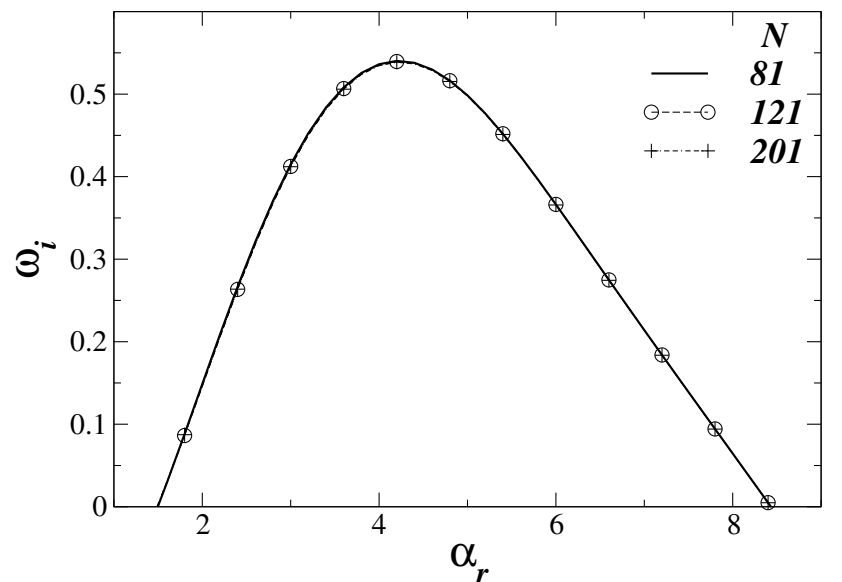

FIG. 2: The effect of increasing the order of Chebyshev polynomials, $N$, on the variation of the growth rate, $\omega_{i}$, with $\alpha_{r}$. The parameters are $\mathrm{Re}=500, \mathrm{Sc}=10, h=0.3, q=0.05$ and $m=2$. 
(a)

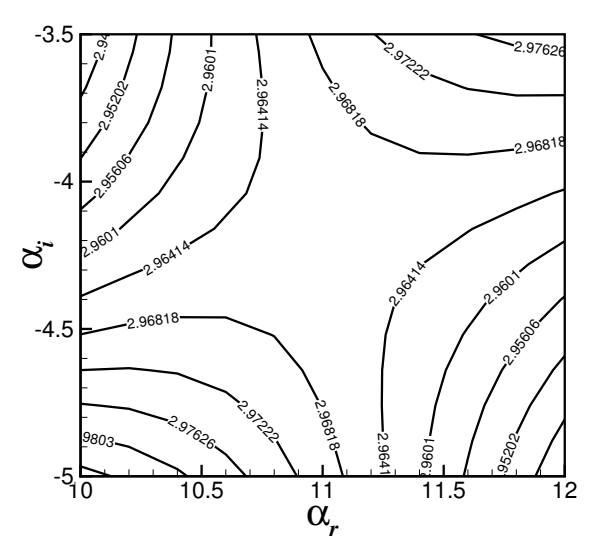

(c)

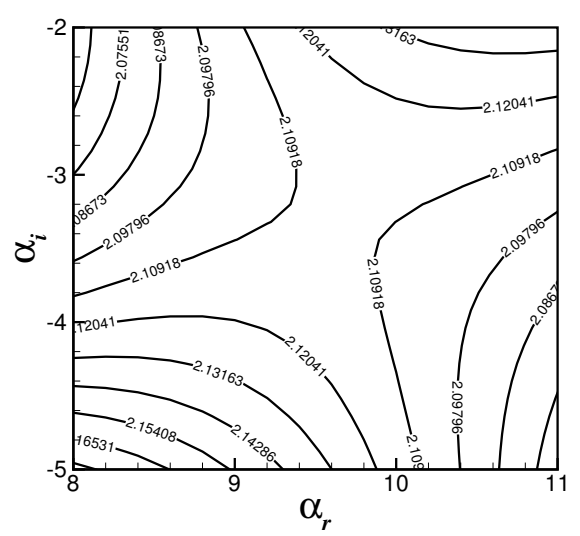

(b)

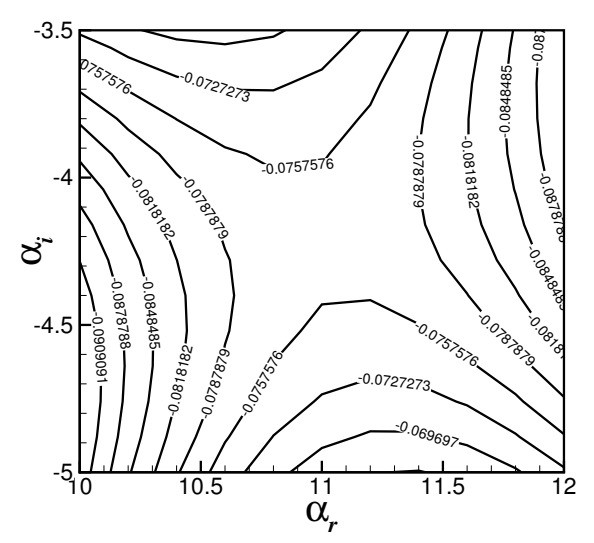

(d)

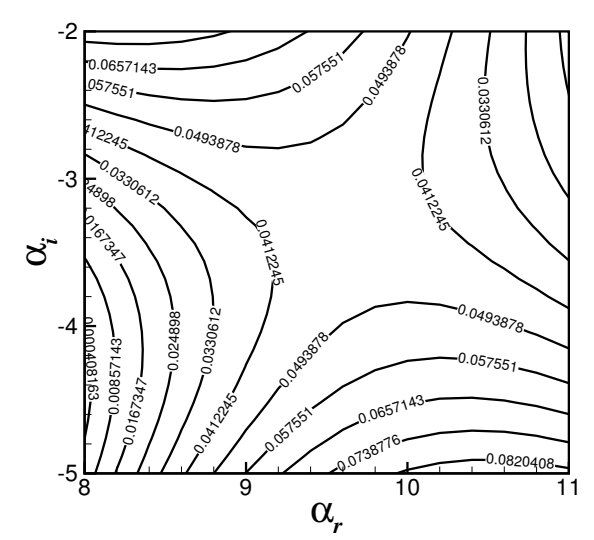

FIG. 3: Isocontours of $\omega_{r}$ and $\omega_{i}$ in the complex wavenumber plane for $m=25$ and $m=40$ are shown in (a) and (b), and (c) and (d), respectively. The value of $\omega$ at the saddle point is $2.966-0.076 i$ and $2.110+0.045 \mathrm{i}$ for $m=25$ and $m=40$, respectively. The rest of the parameter values are $\operatorname{Re}=500, \mathrm{Sc}=50, h=0.3$ and $q=0.05$. 
(a)

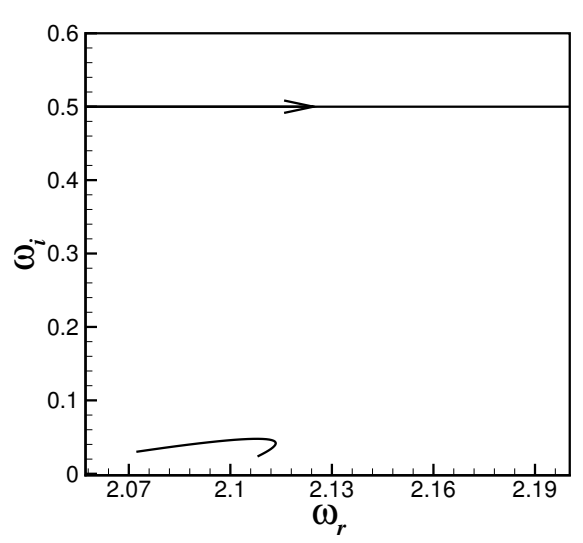

(c)

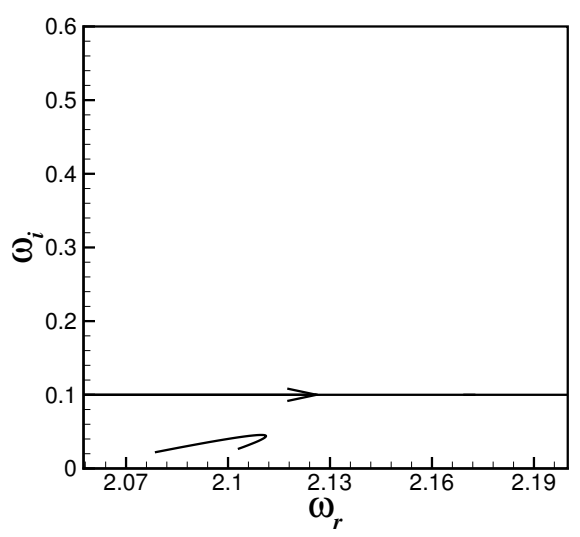

(e)

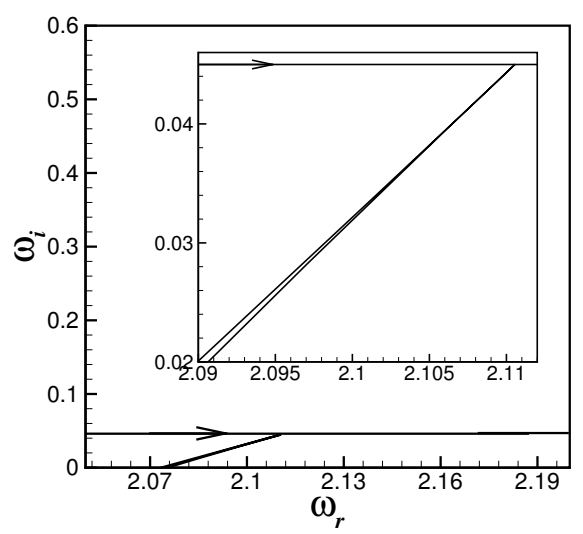

(b)

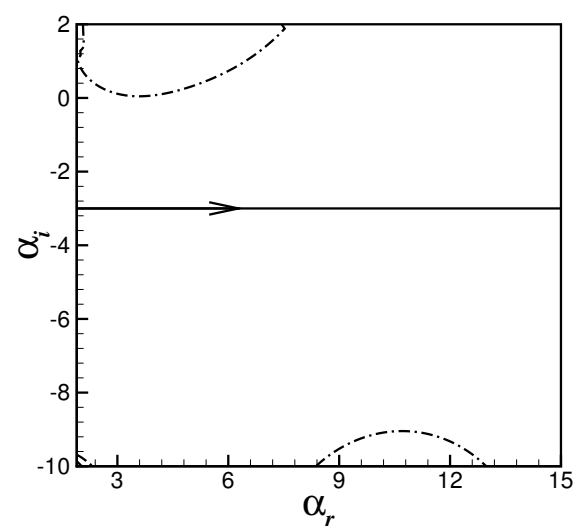

(d)

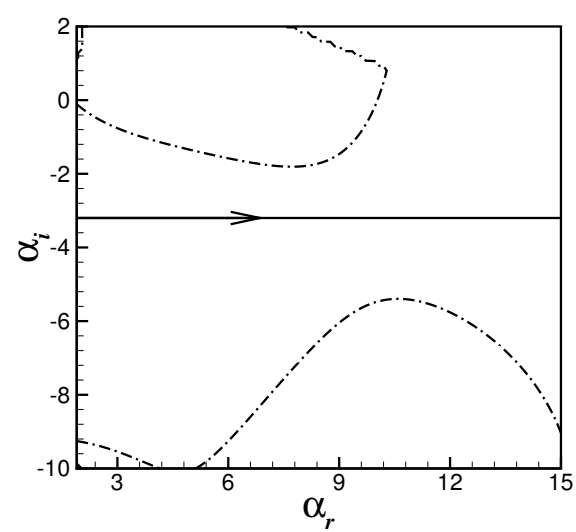

(f)

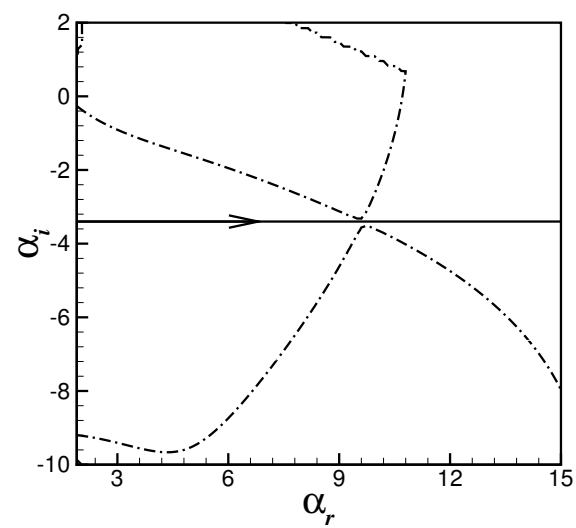

FIG. 4: Contour deformation procedure following the Briggs method [54] with $m=40$ showing the coalescence of two spatial branches initially present in the upper and lower halves of the complex $\alpha$ plane. Panels (a), (c) and (e) show the effect of contour deformation in the complex $\omega$ plane, while (b), (d) and (f) show the analogous effect in the complex $\alpha$ plane. The rest of the parameters are the same as in Fig. 3. The inset in panel (e) shows details of the branch point in the complex $\omega$-plane. 
(a)

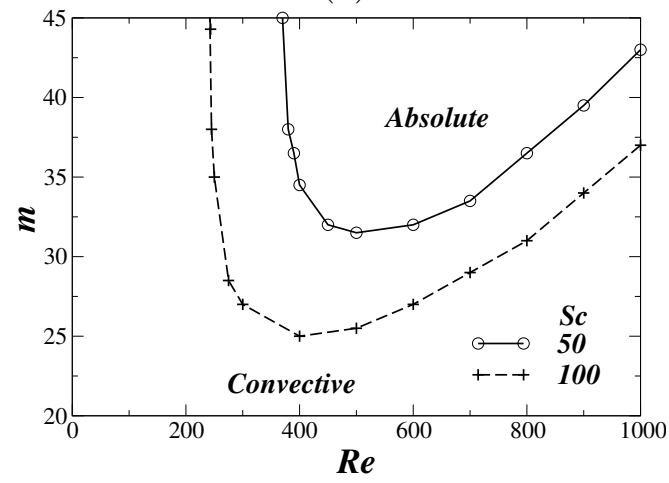

(b)

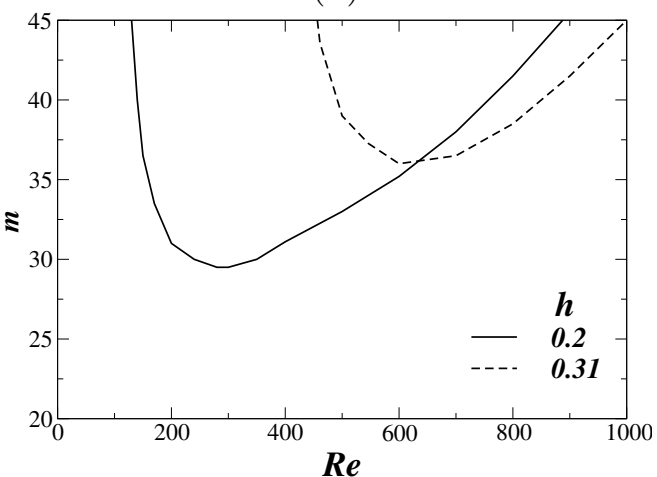

FIG. 5: Stability diagram showing the regions of convective and absolute instability in $m-\operatorname{Re}$ space. (a) Effect of Sc with $h=0.3$; (b) effect of $h$ with Sc $=50$. In both panels, $q=0.05$. 
(a)

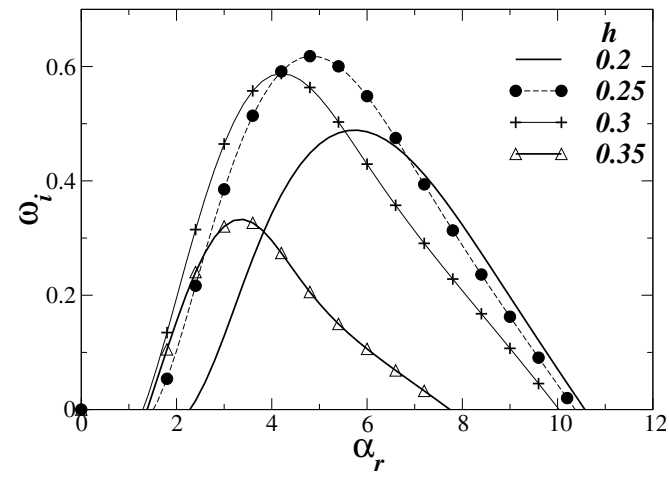

(b)

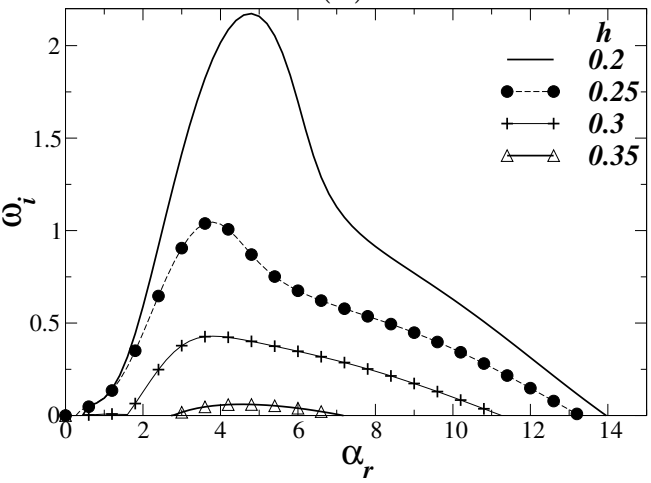

FIG. 6: The effect of $h$ on the dispersion curves, $\omega_{i}$ vs. $\alpha_{r}$, for $m=2$ and $m=40$, shown in (a) and (b), respectively. The rest of the parameters are $\mathrm{Re}=500, \mathrm{Sc}=50$ and $q=0.05$. 
(a)

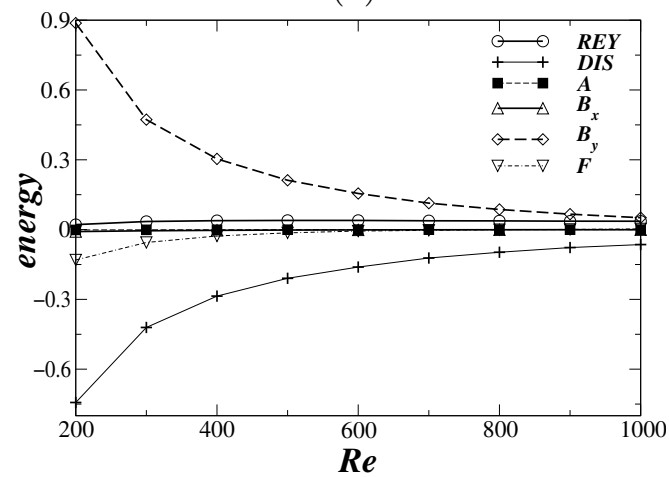

(b)

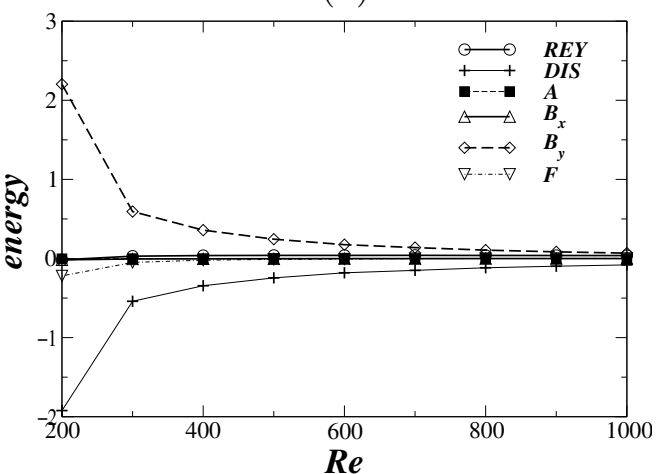

FIG. 7: Variations of the different contributions to the rate of change of the disturbance kinetic energy with Re with $S c=100$ and $m=30$, and $S c=50$ and $m=40$, shown in (a) and (b), respectively. The rest of the parameter values are $h=0.3$ and $q=0.05$. 
(a)

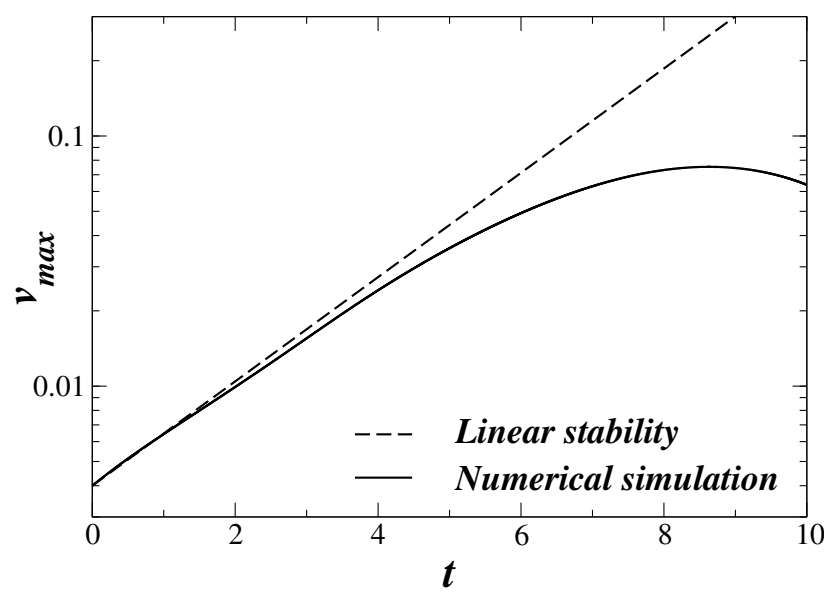

(b)

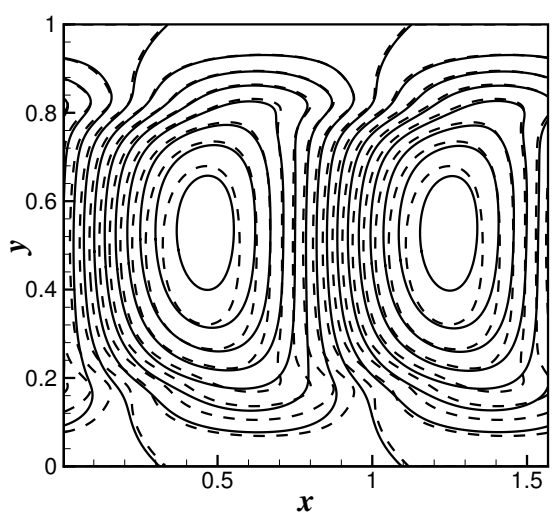

(c)

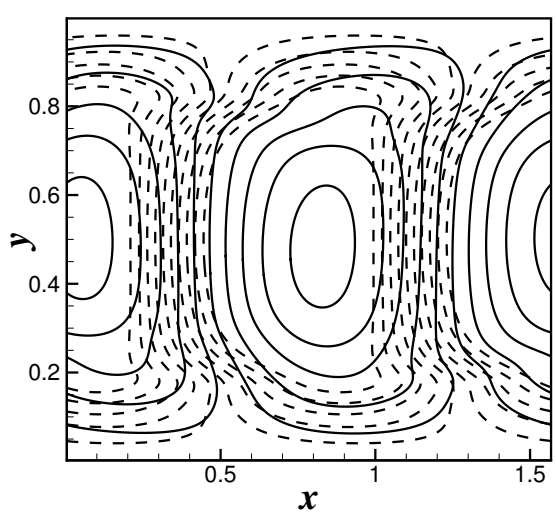

FIG. 8: (a) Comparison of the temporal evolution of the vertical velocity perturbation from the linear stability analysis with that obtained from the numerical simulation with 256 points in the $x$ and $y$ directions for $\alpha_{r}=4$. The direct numerical simulation result with 361 points in the $x$ and $y$ directions is virtually indistinguishable from the one shown in this figure. Comparison of the contour of the vertical velocity perturbation from the linear stability analysis (dashed line) with that from numerical simulations (solid line) at $t=1$, (b), and $t=6$, (c). The rest of the parameters are $\operatorname{Re}=500, \mathrm{Sc}=10, m=2, h=0.3$ and $q=0.05$. 
(a)

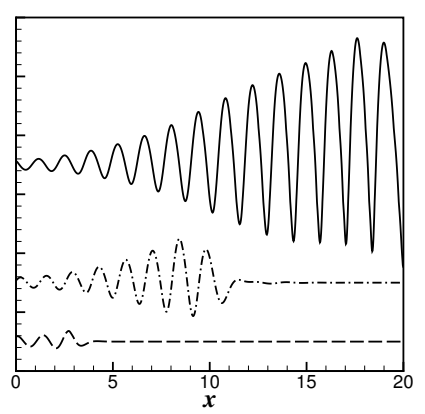

(c)

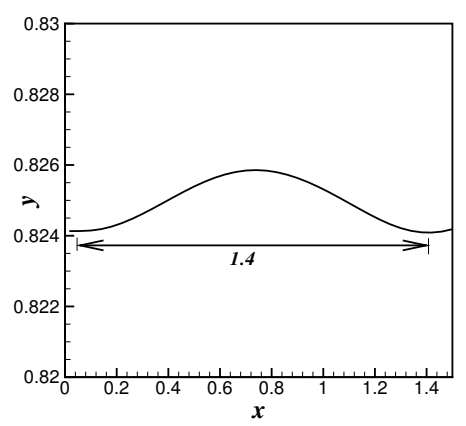

(e)

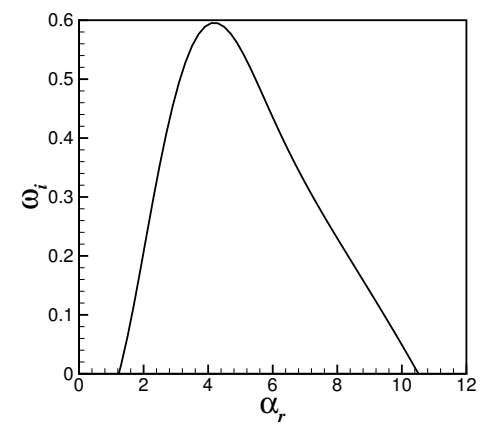

(b)

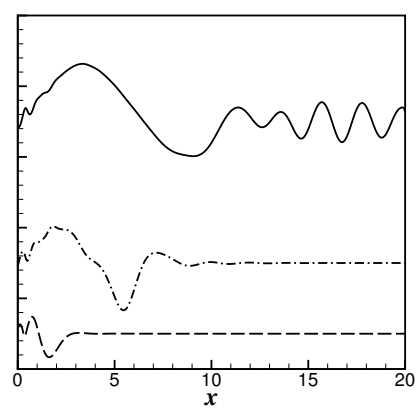

(d)

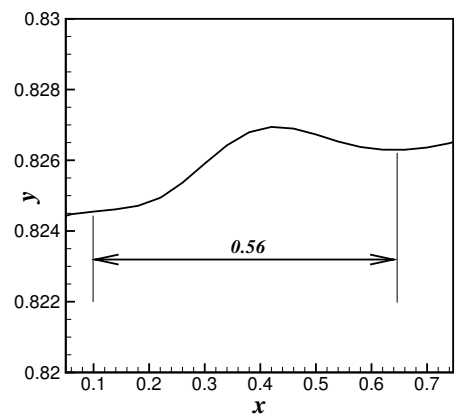

(f)

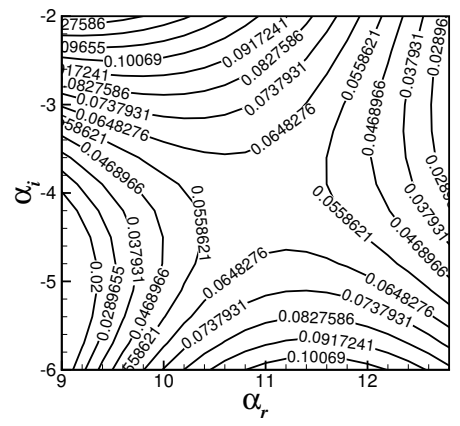

FIG. 9: Spatio-temporal evolution of the contours of $c=0.5$ for $m=2$, (a), and $m=30$, (b); solutions are shown at $t=4$ (dotted lines), $t=12$ (dash-dotted line) and $t=20$ (solid line). For clarity of presentation, the dash-dotted and solid lines in panels (a) and (b) are displaced by 0.01 and 0.03, respectively, from their original position in the vertical axis. The rest of the parameter values are $\operatorname{Re}=500, \mathrm{Sc}=100, h=0.3$ and $q=0.05$. Enlarged views of the $c=0.5$ contour near the channel inlet for $m=2$ and $m=30$, shown in (c) and (d), respectively. (e) and (f) show the dispersion curve for $m=2$ and the contour of $\omega_{i}$ in the complex $\alpha$-plane. The estimates of the disturbance wavelength shown panels (c) and (d) correspond approximately to those of the most dangerous temporal mode and the mode associated with the pinch point singularity at the saddle point in panels (e) and (f), respectively. 


$$
t=10
$$

$$
t=20
$$

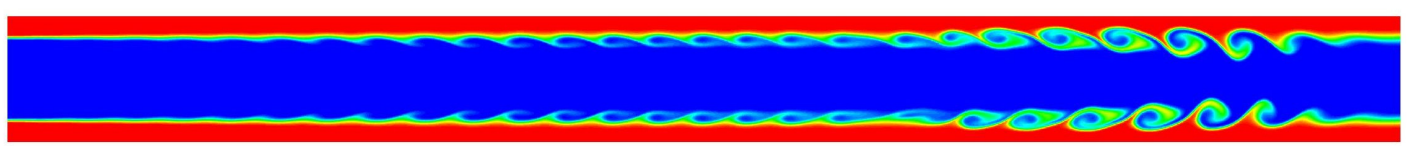

$$
t=30
$$

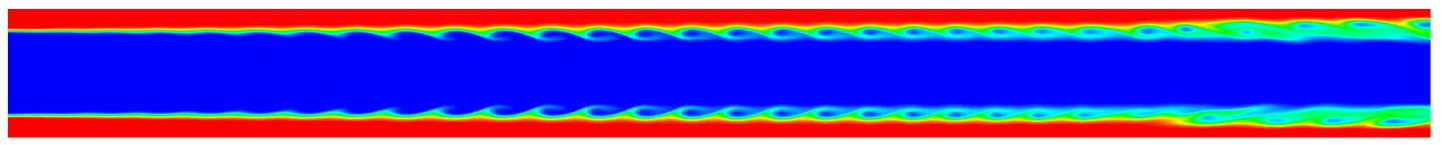

$$
t=40
$$

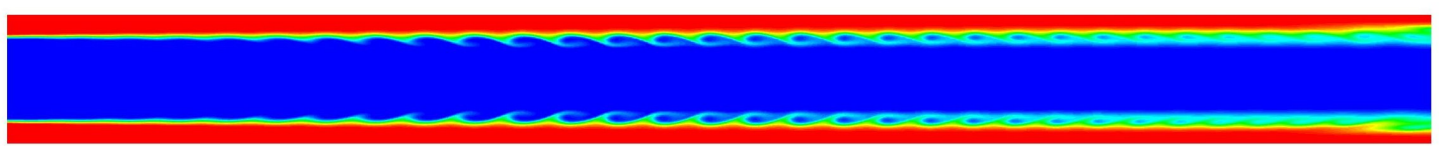

$$
t=50
$$

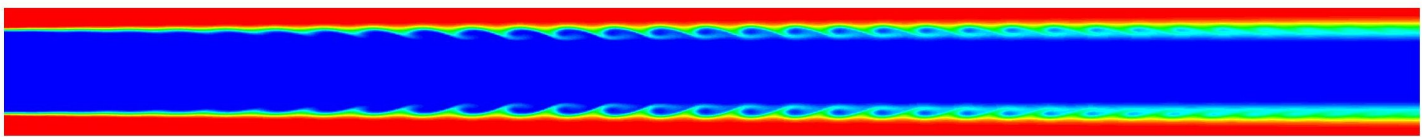

\section{$t=100$}

FIG. 10: Spatio-temporal evolution of the concentration contours for $m=2, \operatorname{Re}=500, \mathrm{Sc}=100$, $h=0.3$, and $q=0.05$. 


$$
t=10
$$

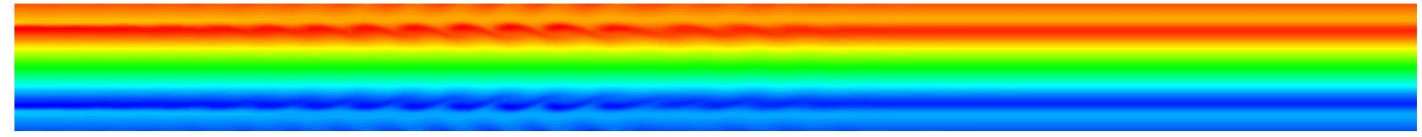

$$
t=20
$$

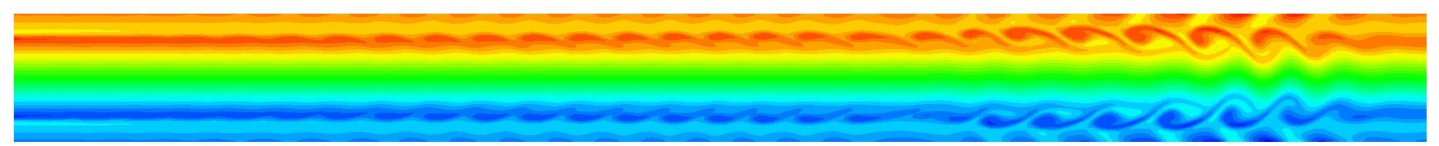

$$
t=30
$$

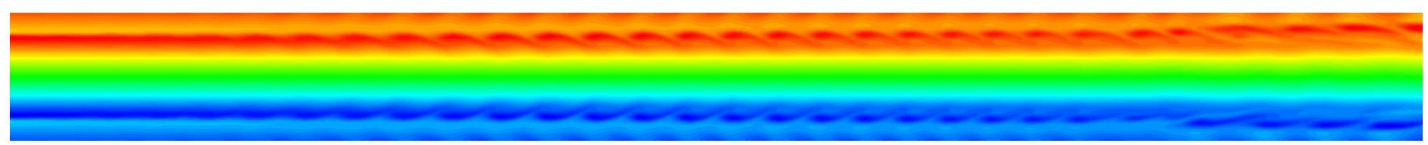

$$
t=40
$$

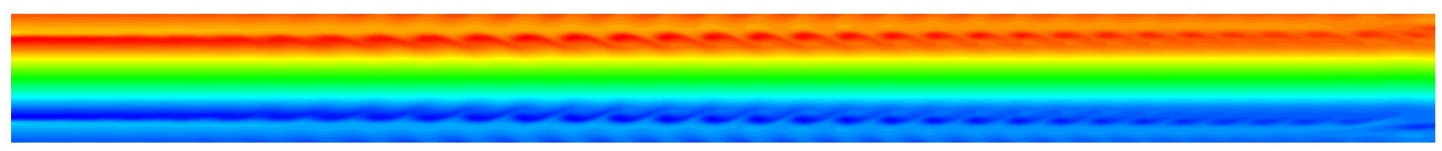

$$
t=50
$$

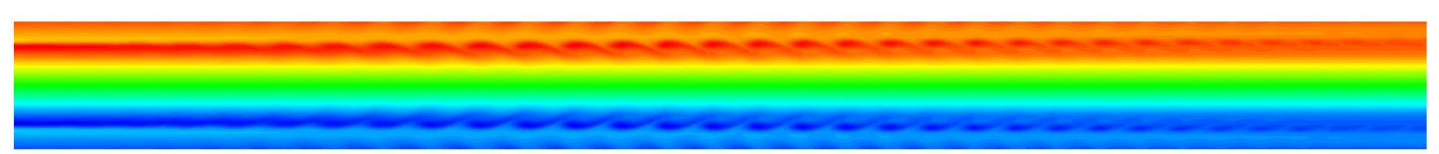

\section{$t=100$}

FIG. 11: Spatio-temporal evolution of the vorticity contours. The parameter values remain unchanged from Fig. 10 


$$
t=20
$$

$$
t=25
$$

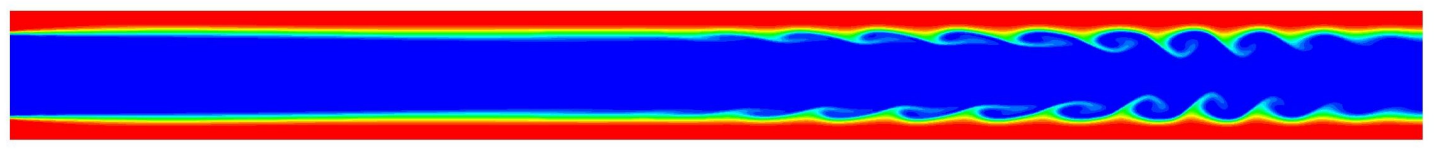

$$
t=30
$$

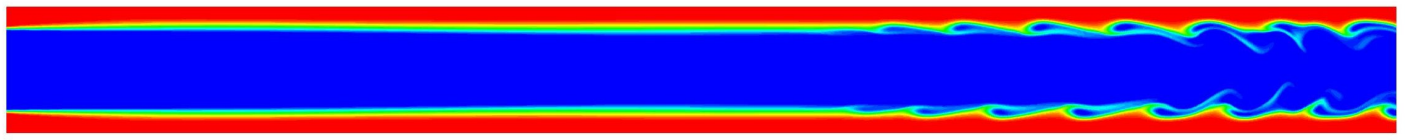

$$
t=40
$$

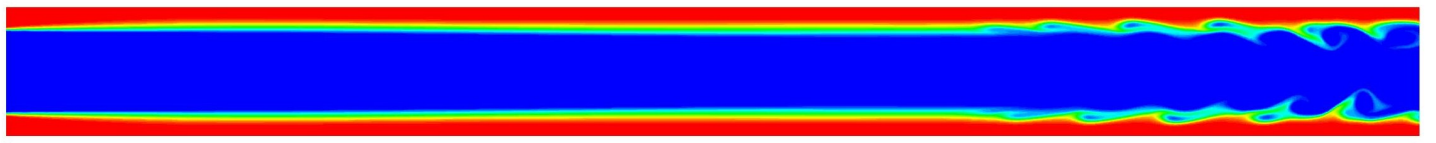

$$
t=50
$$

$$
t=65
$$

FIG. 12: Spatio-temporal evolution of the concentration contours for $m=30$. The rest of the parameter values remain unchanged from Fig. 10. 
(a)

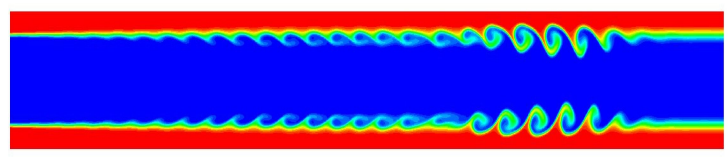

(c)

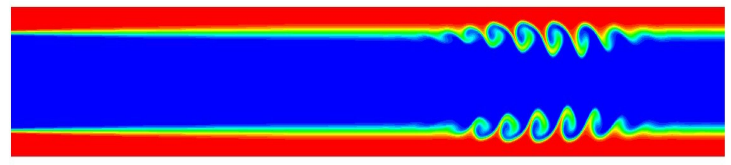

(b)

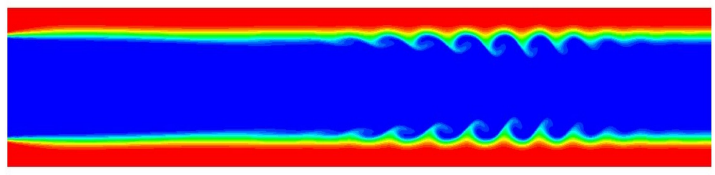

(d)

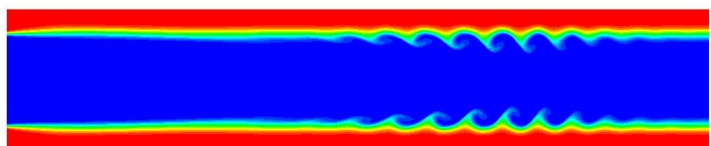

FIG. 13: Concentration contours obtained in the presence and absence of inlet forcing shown in (a) and (b), and (c) and (d), respectively at $t=28$. The results depicted in (a) and (c) were generated with $m=2$, while those in (b) and (d) were obtained with $m=30$. The rest of the parameter values remain unchanged from Fig. 10. 


$$
t=20
$$

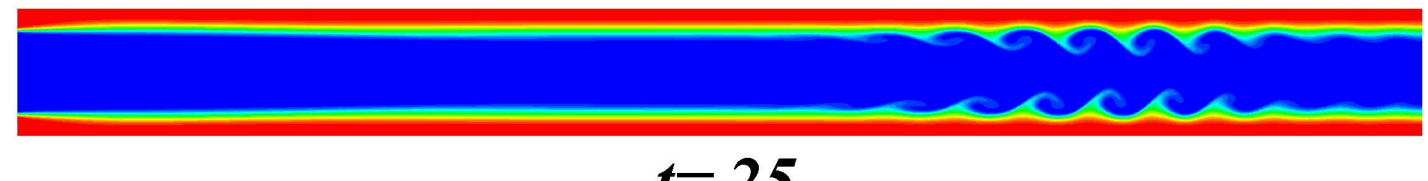

$t=25$

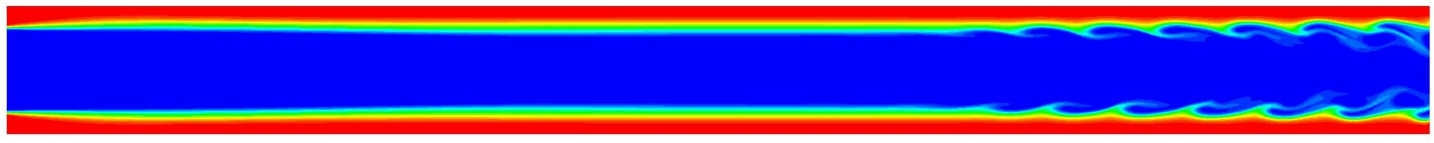

$t=30$

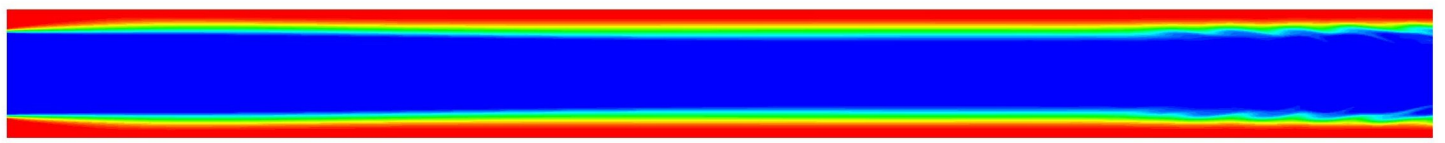

$t=40$

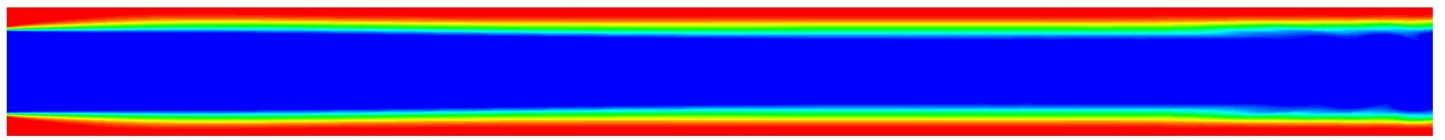

$$
t=45
$$

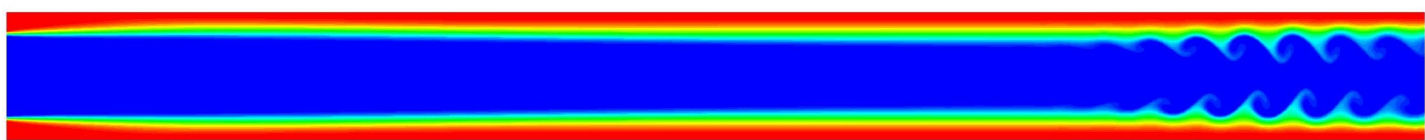

$$
t=50
$$

FIG. 14: Spatio-temporal evolution of the concentration contours for $\mathrm{Sc}=50$. The rest of the parameter values remain unchanged from Fig. 12. 


\section{$t=5$}

$$
t=10
$$

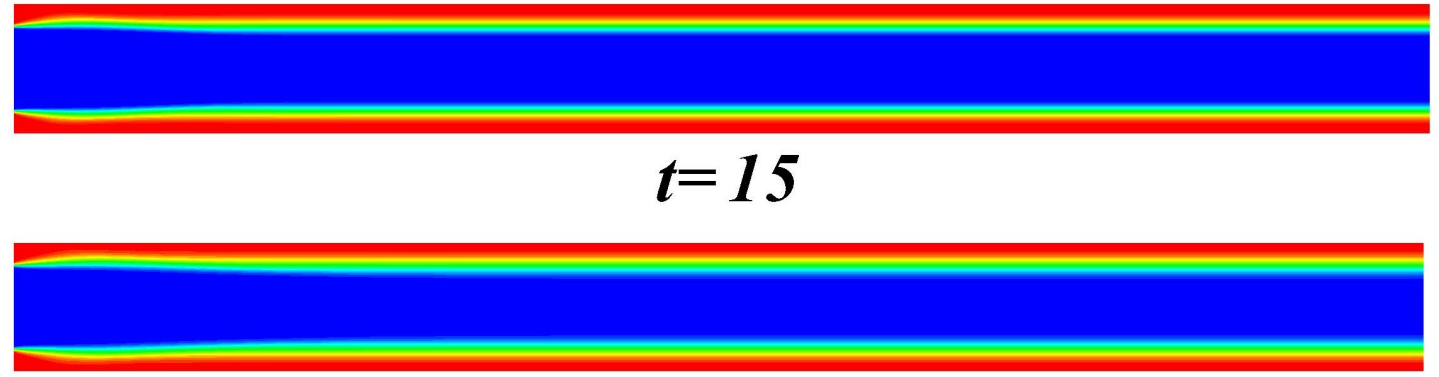

$$
t=20
$$

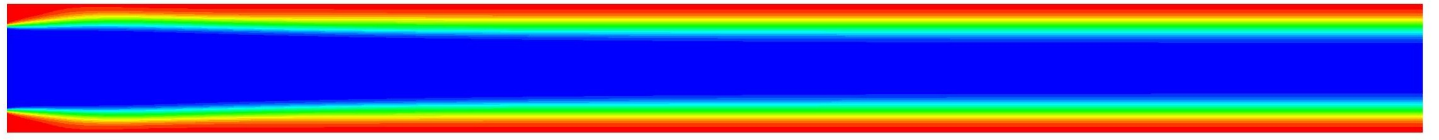

$$
t=30
$$

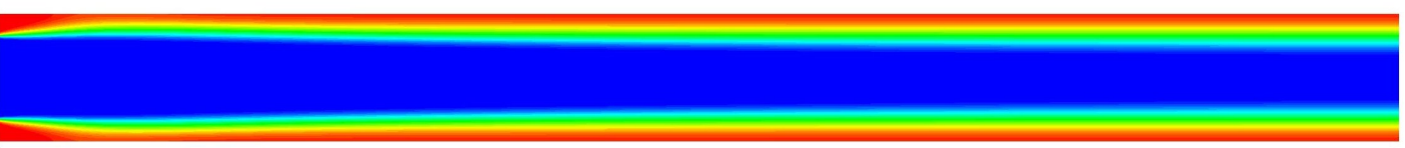

$$
t=45
$$

FIG. 15: Spatio-temporal evolution of the concentration contours for $R e=100$. The rest of the parameter values remain unchanged from Fig. 12. 


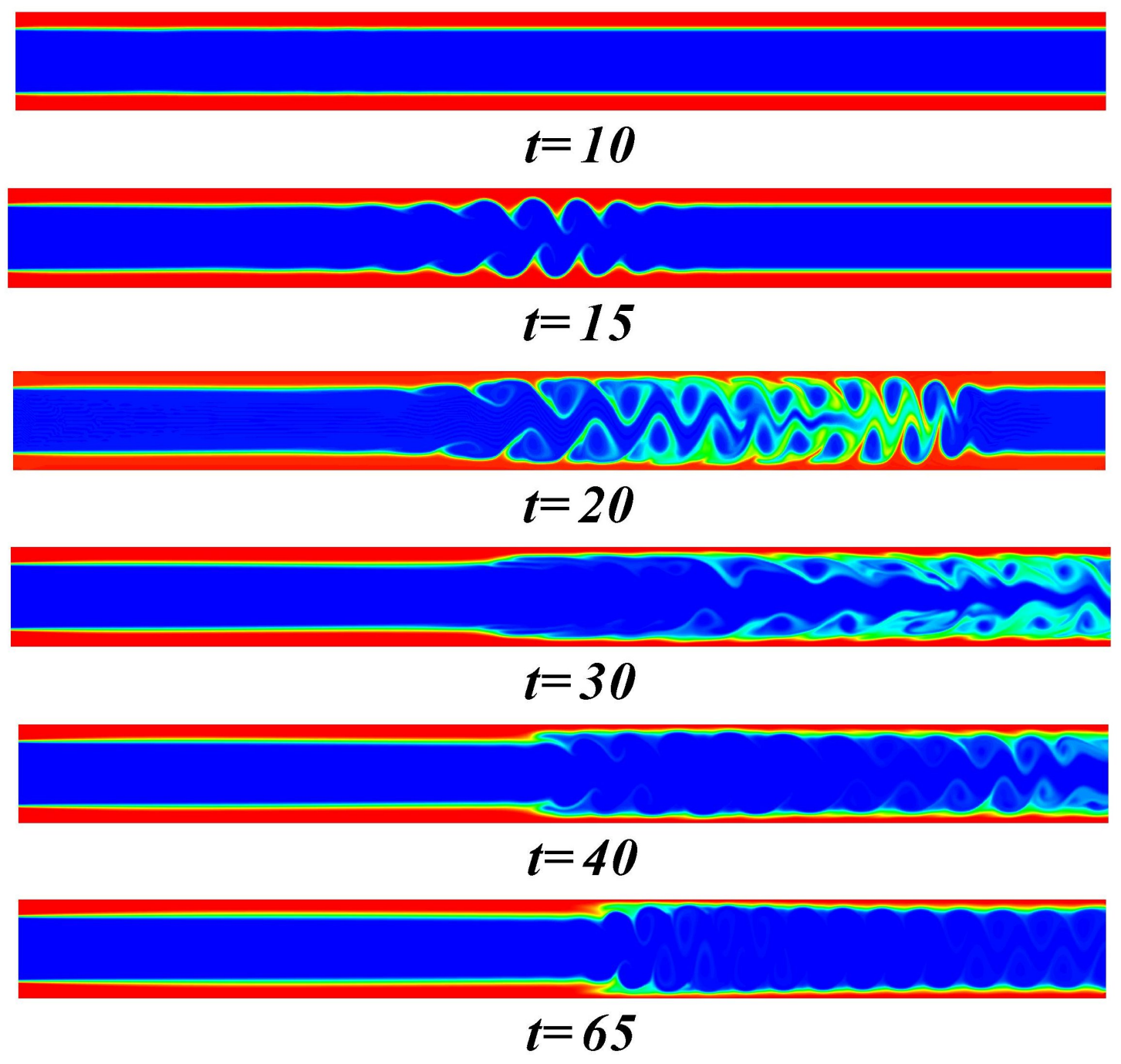

FIG. 16: Spatio-temporal evolution of the concentration contours for $R e=1000$. The rest of the parameter values remain unchanged from Fig. 12. 


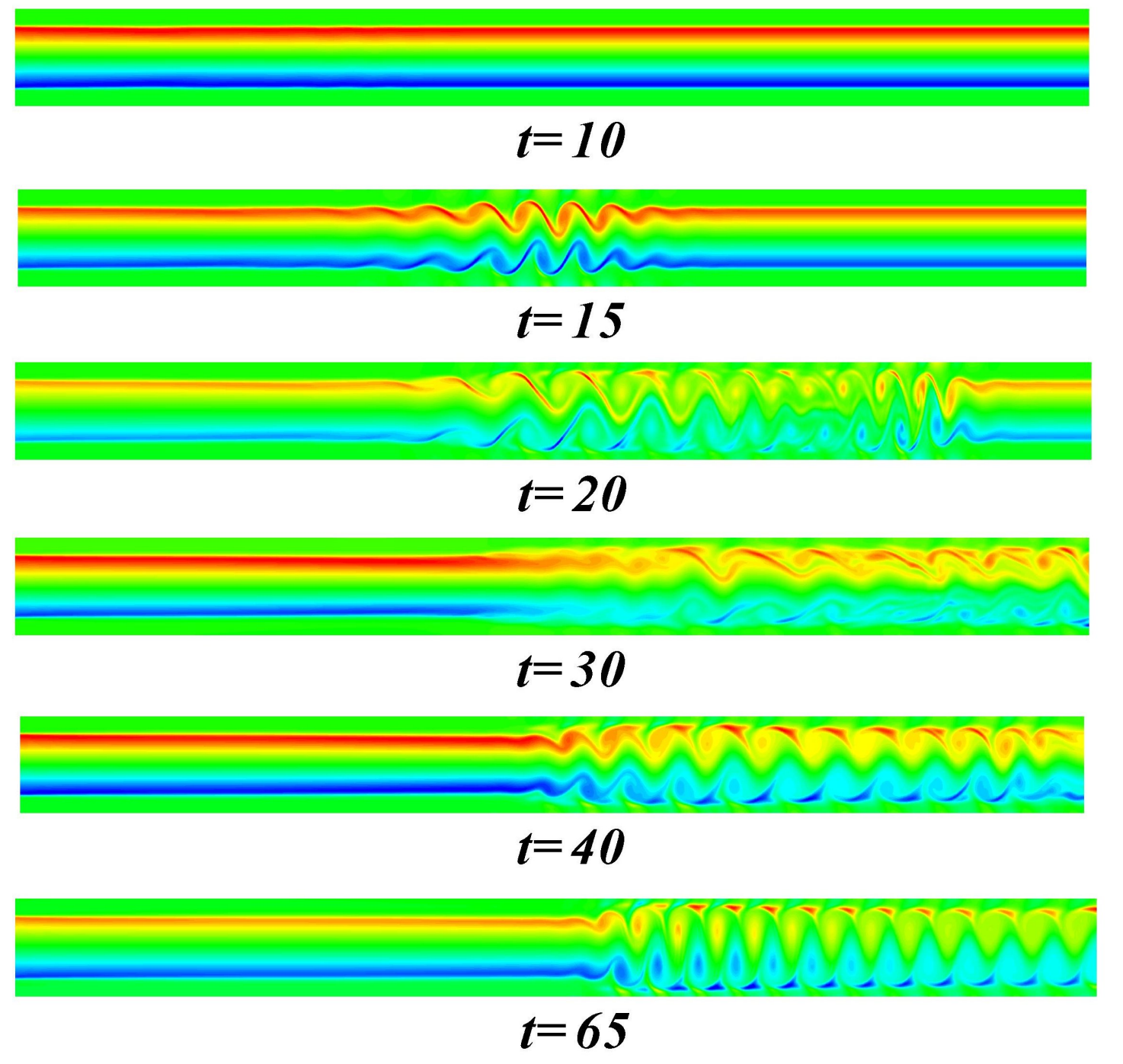

FIG. 17: Spatio-temporal evolution of the vorticity contours for the same parameter values as in Fig. 16 . 


$$
t=2
$$

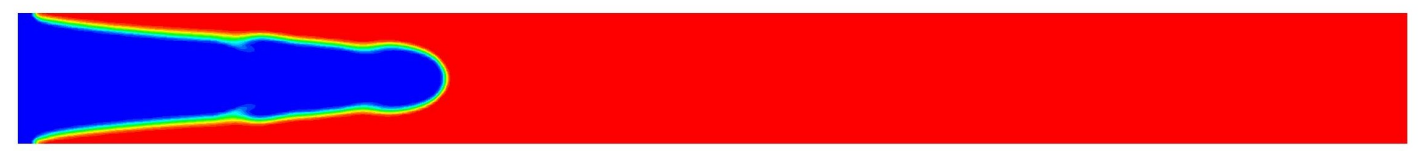

$$
t=8
$$

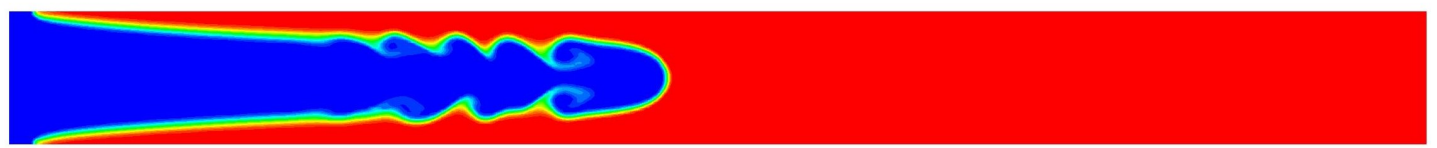

$$
t=12
$$

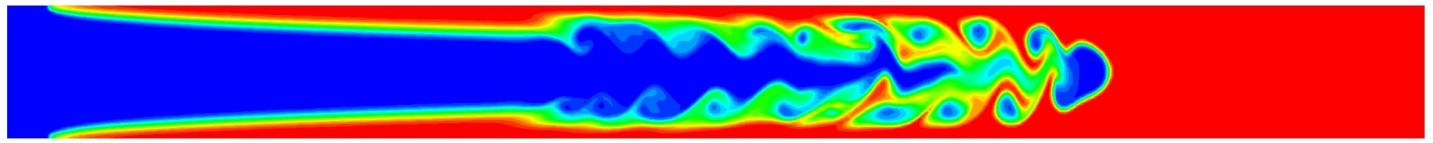

$$
t=20
$$

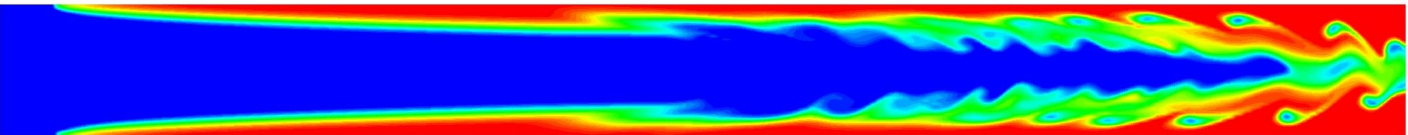

$$
t=26
$$

FIG. 18: Spatio-temporal evolution of the concentration contours for $\operatorname{Re}=500, \mathrm{Sc}=20$ and $m=10$. 
(a)

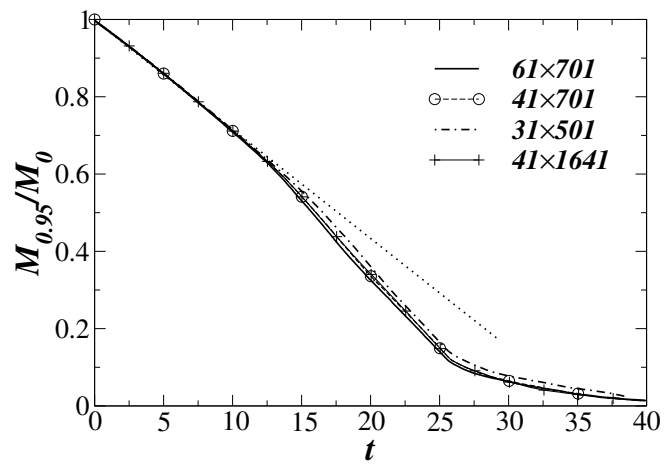

(b)

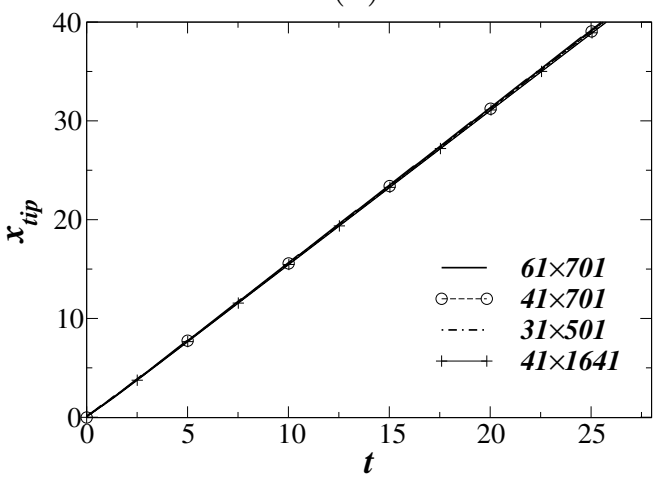

FIG. 19: Mass fraction of the displaced fluid $M_{0.95} / M_{0}$, (a), and temporal evolution of the position of the leading front separating the two fluids, $x_{\text {tip }}$, (b), obtained using different mesh densities for the same parameters as in Fig. 18. The dashed line of constant slope has been included in (a) to demonstrate the constant displacement rate in the flow regime dominated by the unperturbed penetration of a finger of the less viscous fluid into the more viscous one. 


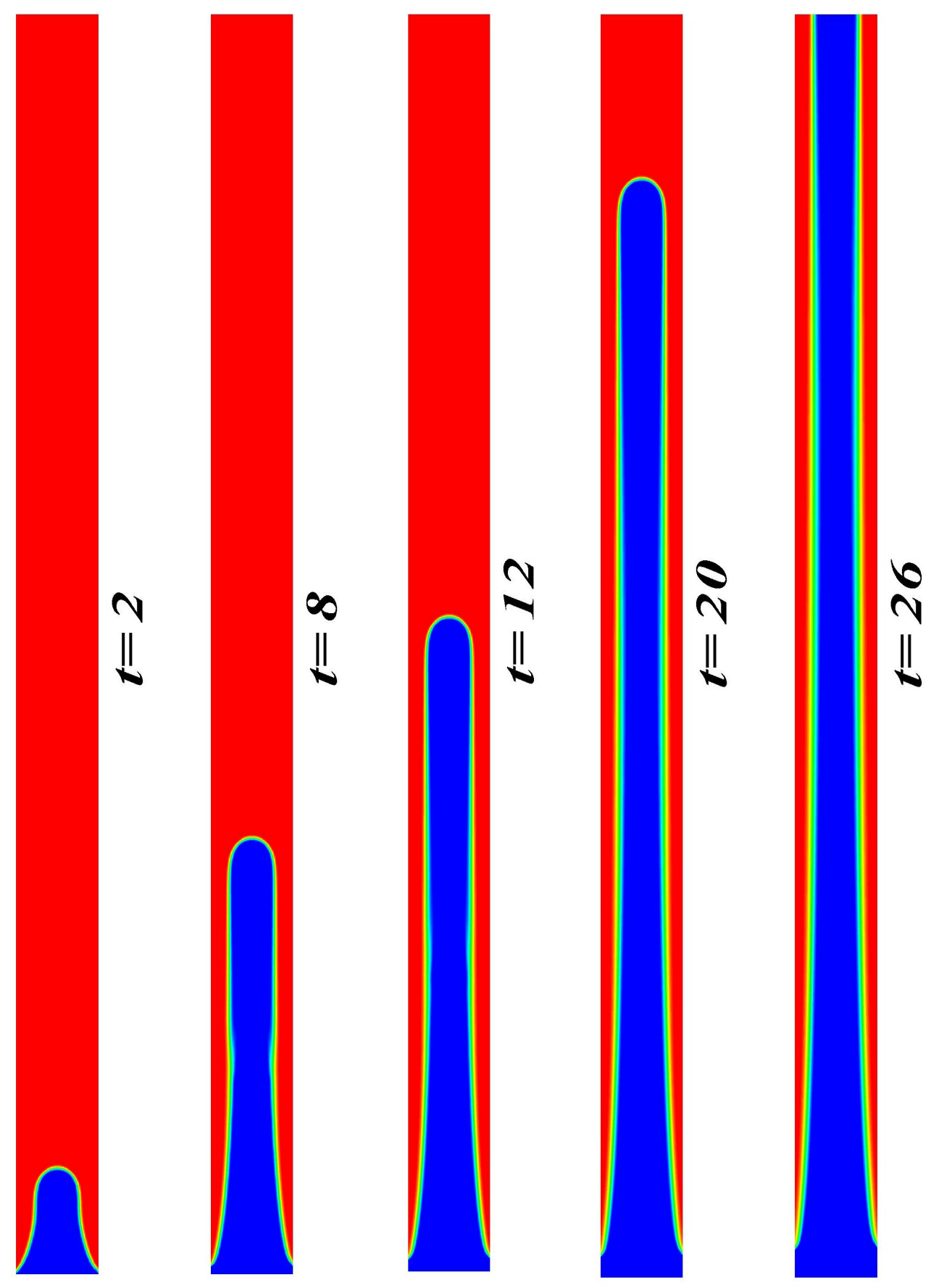

FIG. 20: Spatio-temporal evolution of the concentration contours for $\mathrm{Re}=100, \mathrm{Sc}=100$ and $m=30$. 


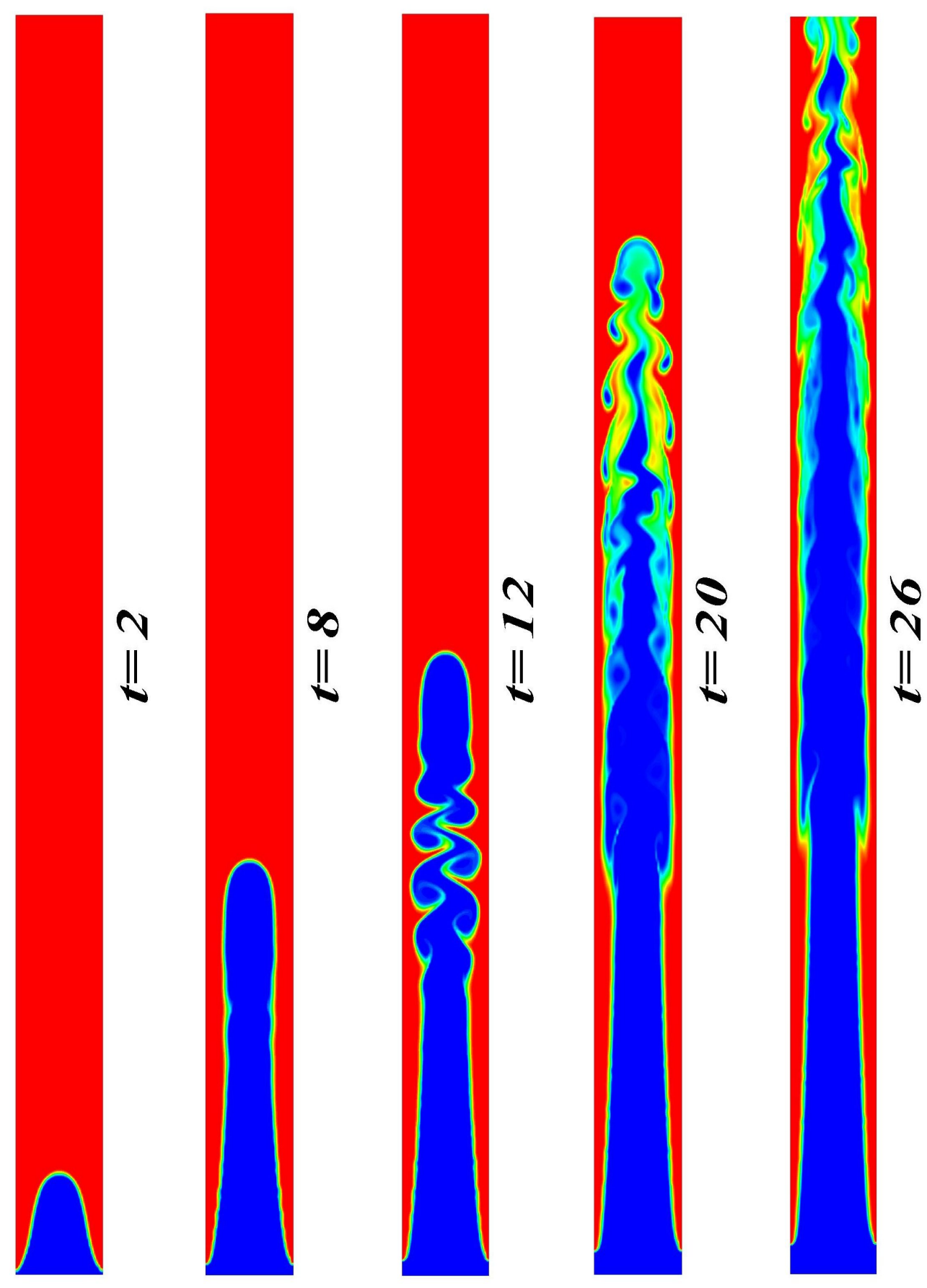

FIG. 21: Spatio-temporal evolution of the concentration contours for $R e=500$. The rest of the parameter values remain unchanged from Fig. 20. 


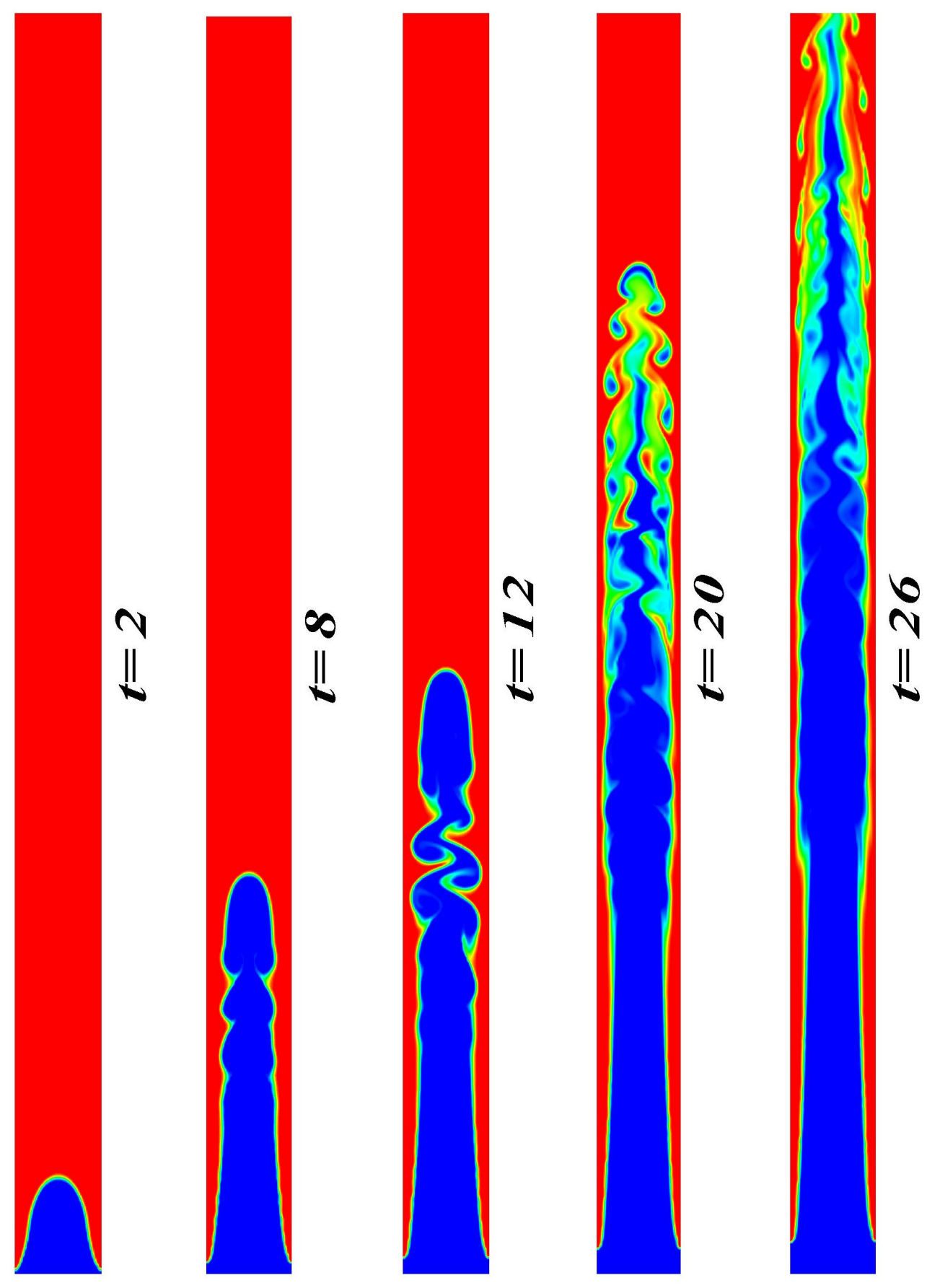

FIG. 22: Spatio-temporal evolution of the concentration contours for $R e=1000$. The rest of the parameter values remain unchanged from Fig. 20. 


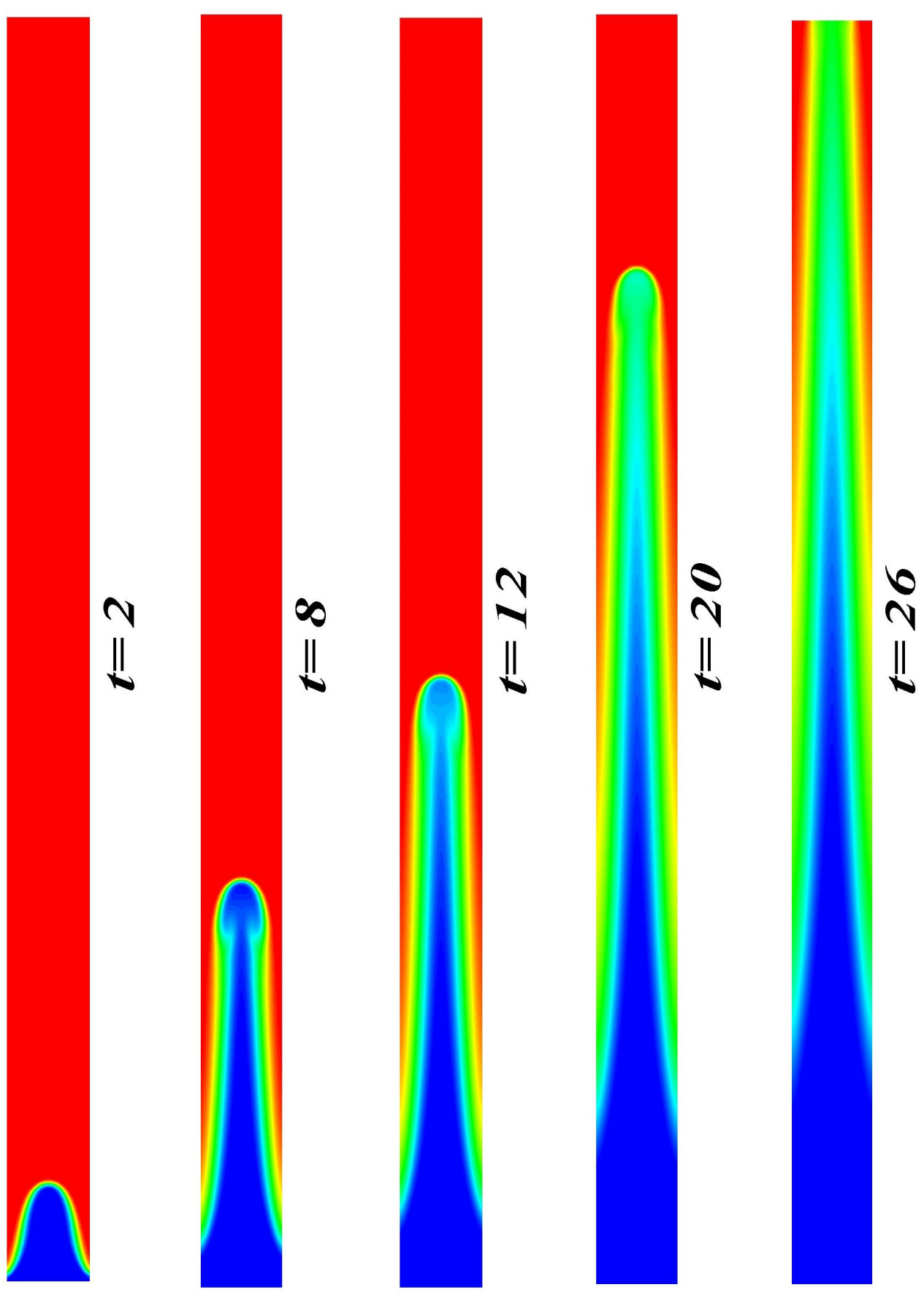

FIG. 23: Spatio-temporal evolution of the concentration contours for $\mathrm{Sc}=1, \operatorname{Re}=500$ and $m=25$. 


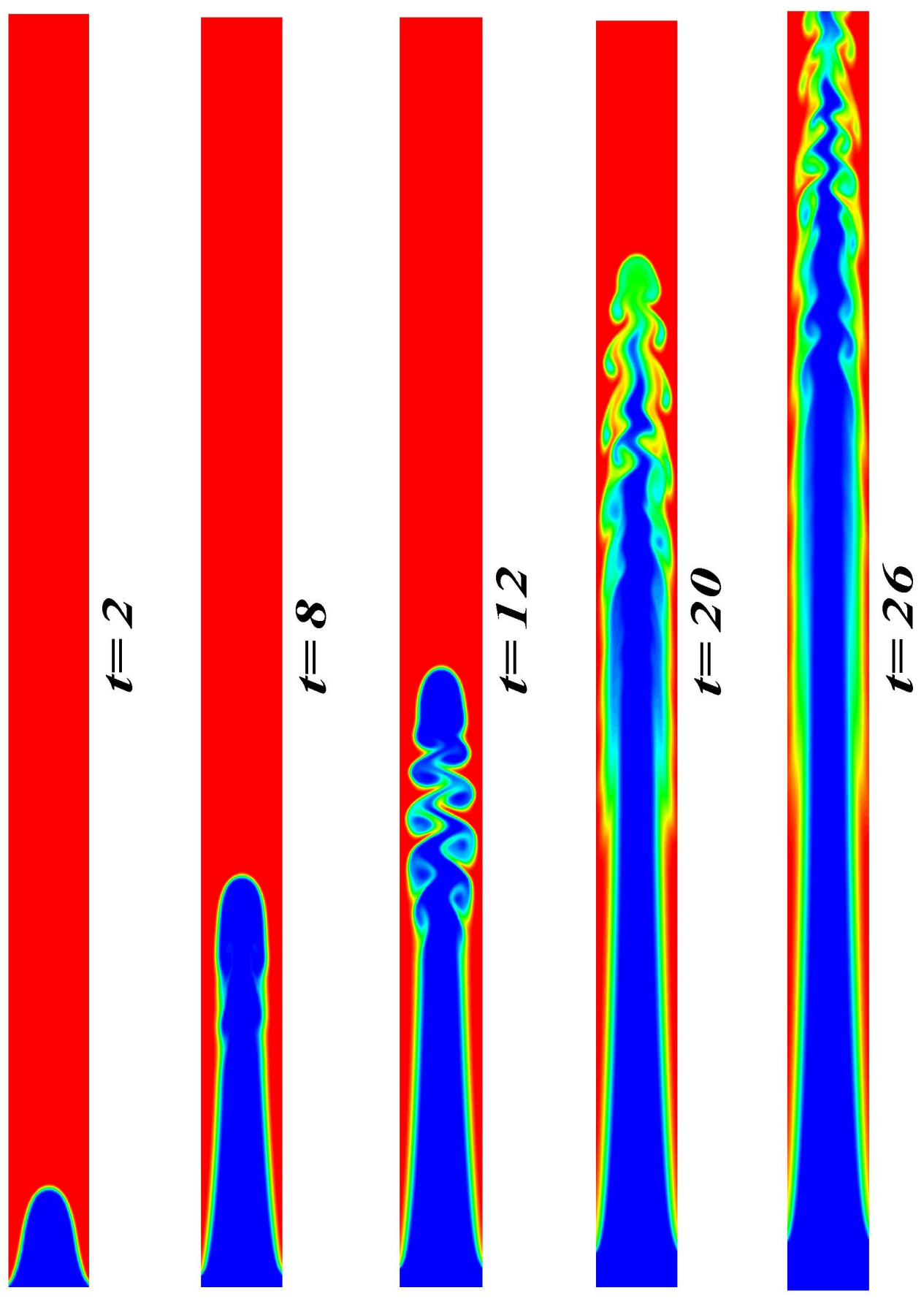

FIG. 24: Spatio-temporal evolution of the concentration contours for $\mathrm{Sc}=10$. The rest of the parameter values remain unchanged from Fig. 23. 


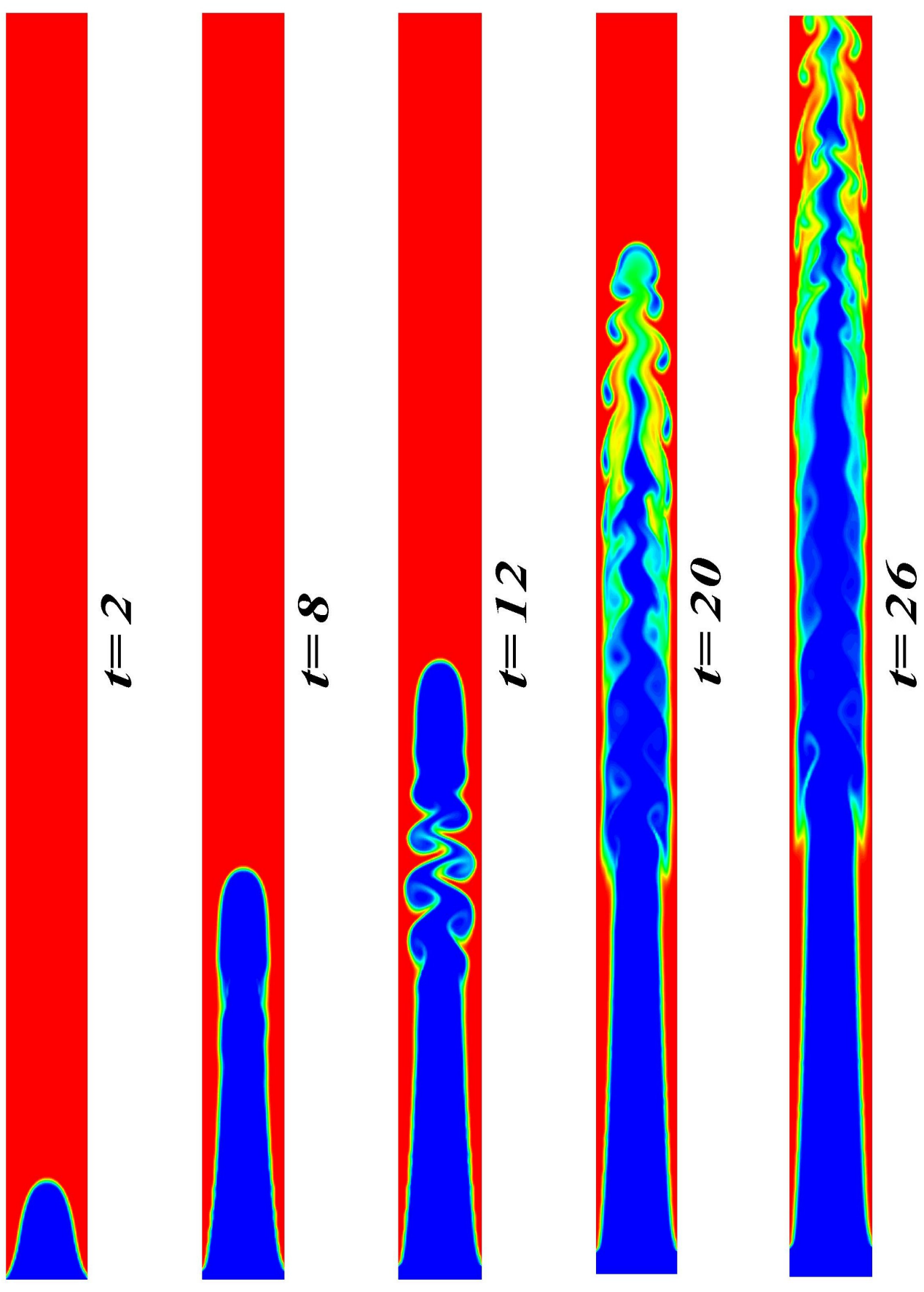

FIG. 25: Spatio-temporal evolution of the concentration contours for $\mathrm{Sc}=100$. The rest of the parameter values remain unchanged from Fig. 23. 


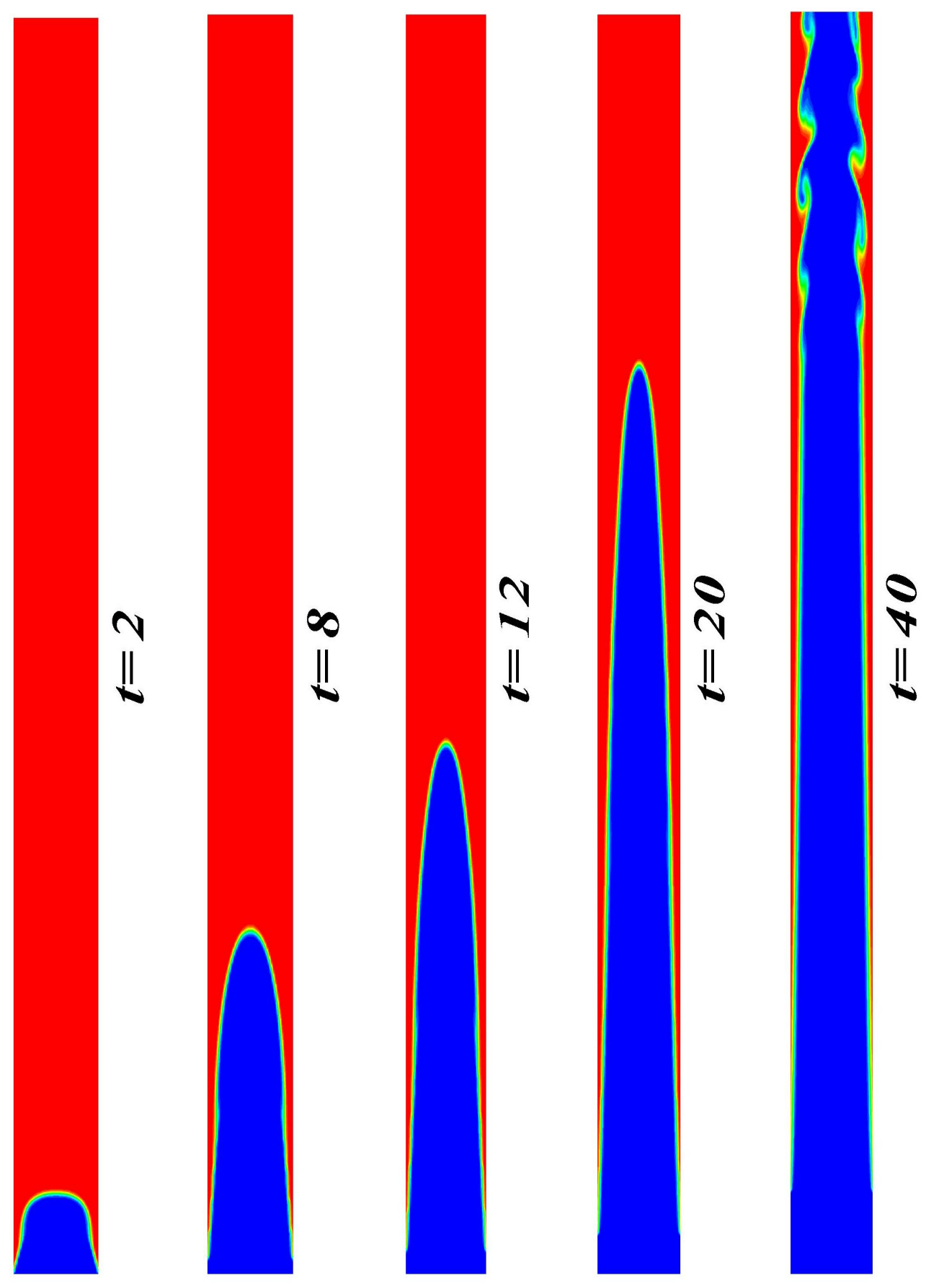

FIG. 26: Spatio-temporal evolution of the concentration contours for $m=2, \operatorname{Re}=500$ and $\mathrm{Sc}=100$. 


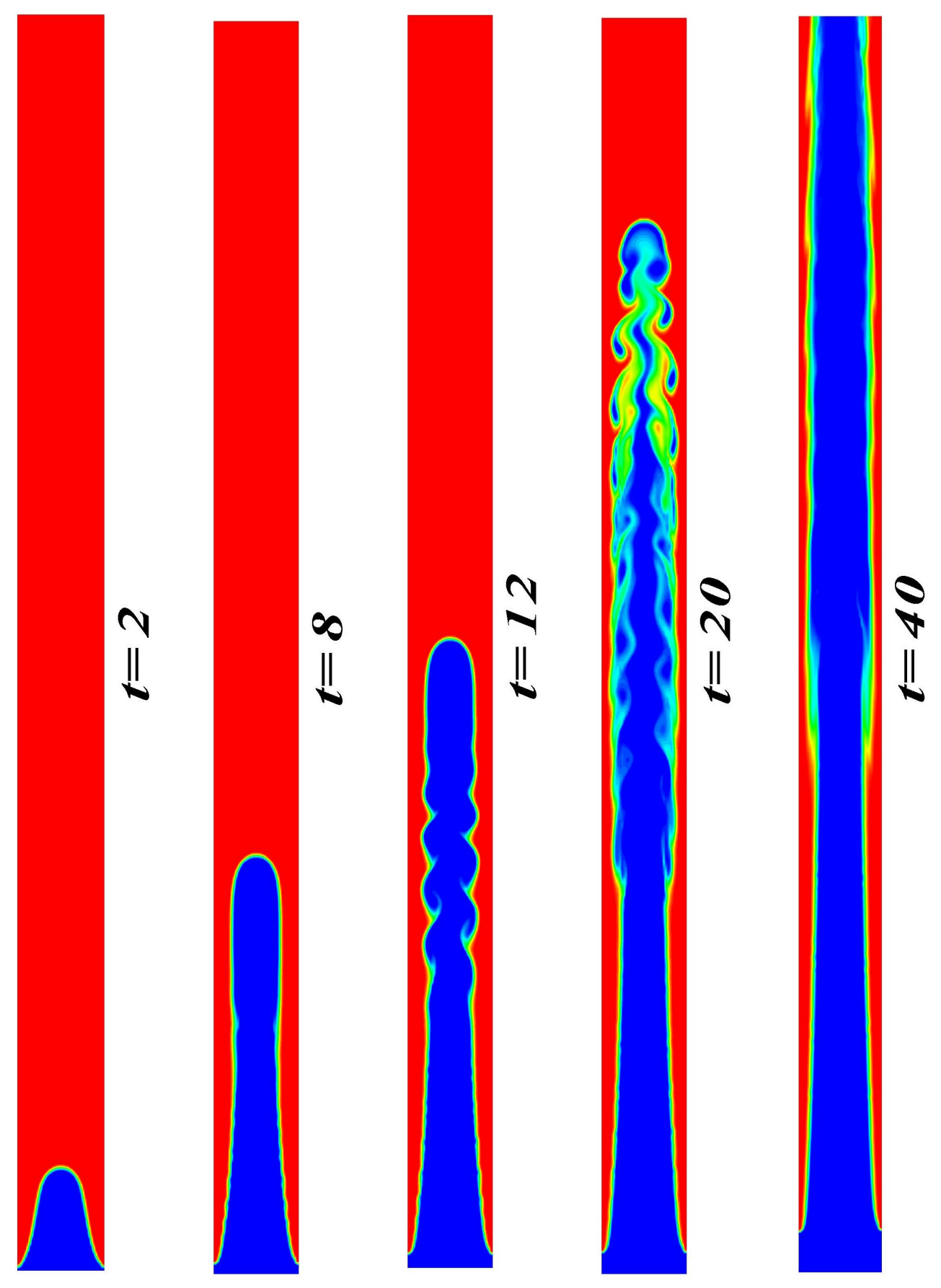

FIG. 27: Spatio-temporal evolution of the concentration contours for $m=40$. The rest of the parameter values remain unchanged from Fig. 26. 
(a)

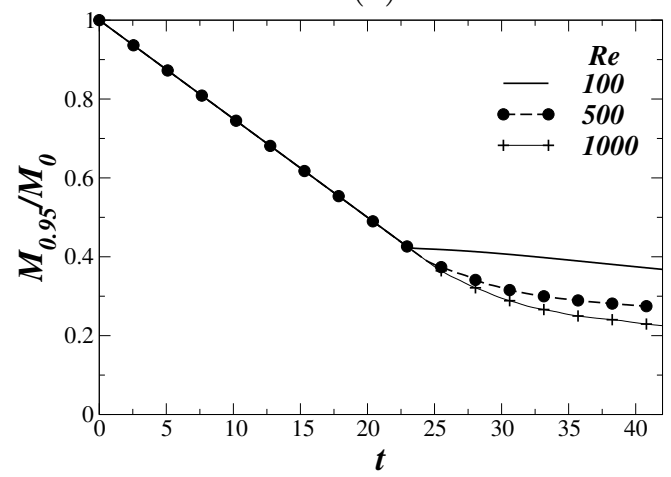

(c)

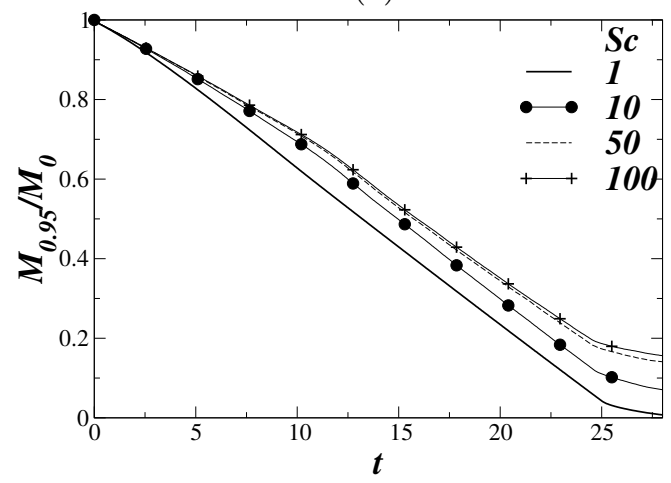

(e)

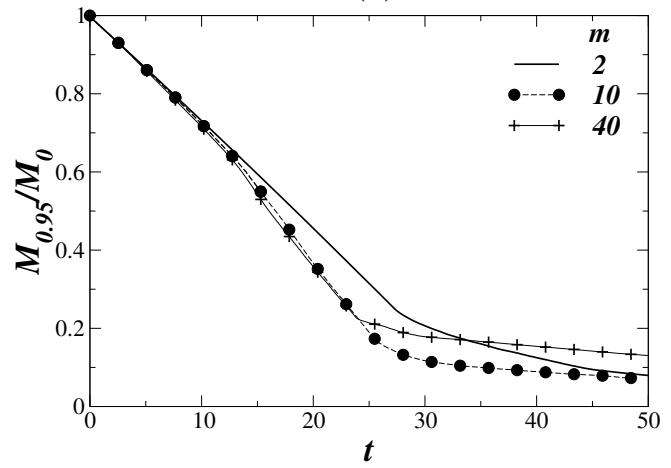

(b)

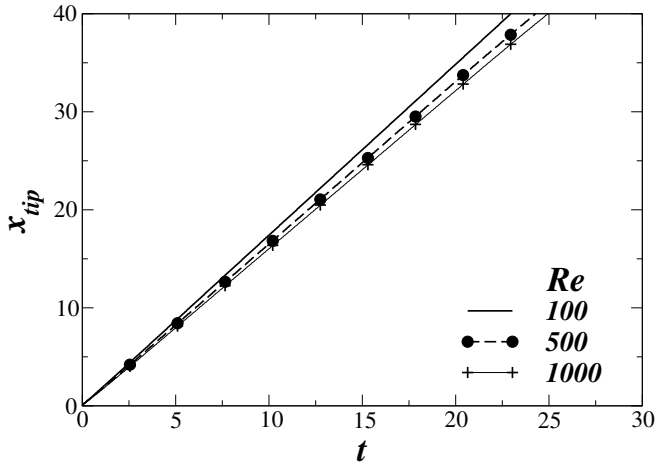

(d)

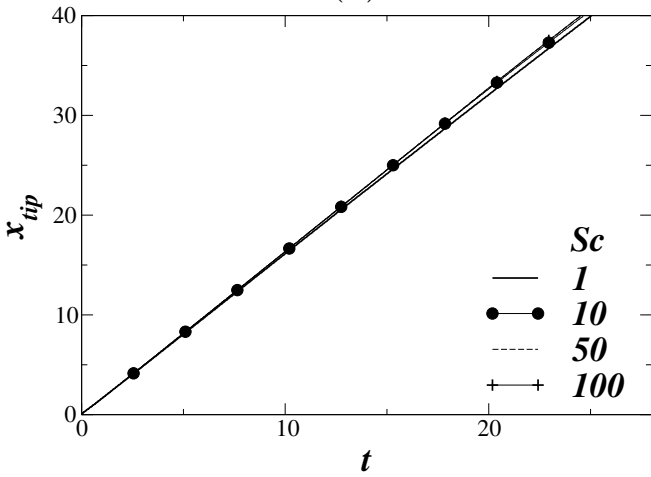

(f)

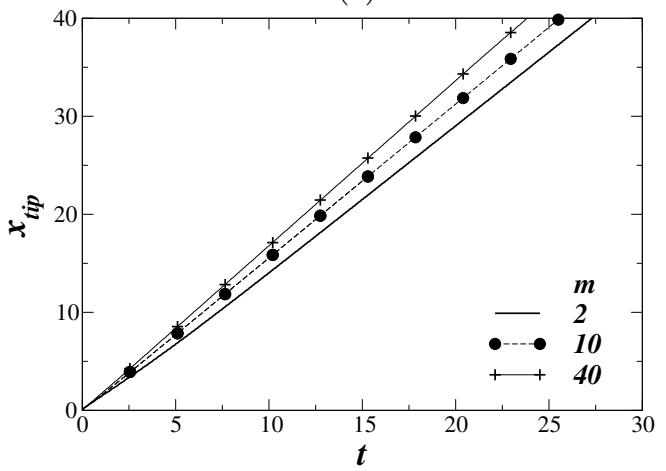

FIG. 28: Effect of Re, Sc and $m$ on the mass fraction of the displaced fluid ' 1 ' and the temporal evolution of the position of the leading front separating the two fluids, $x_{\text {tip }}$ shown in (a),(c), (e), and (b), (d), (f), respectively. The rest of the parameter values are Sc $=100$ and $m=30$ in (a) and (b); Re $=500$ and $m=25$ in (c) and (d); and $\mathrm{Re}=500$ and $\mathrm{Sc}=100$ in (e) and (f). 